\title{
Article \\ New Concepts of Vertex Covering in Cubic Graphs with Its Applications
}

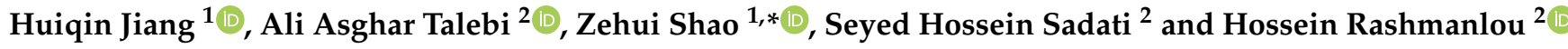 \\ 1 Institute of Computing Science and Technology, Guangzhou University, Guangzhou 510006, China; \\ 1111906006@e.gzhu.edu.cn \\ 2 Department of Mathematics, University of Mazandaran, 4741613534 Babolsar, Iran; \\ a.talebi@umz.ac.ir (A.A.T.); s.sadati02@umail.umz.ac.ir (S.H.S.); rashmanlou.1987@gmail.com (H.R.) \\ * Correspondence: zshao@gzhu.edu.cn
}

Citation: Jiang, H.; Talebi, A.A.; Shao, Z.; Sadati, S.H.; Rashmanlou, H. New Concepts of Vertex Covering in Cubic Graphs with Its Applications.

Mathematics 2022, 10, 307. https:// doi.org/10.3390/math10030307

Academic Editor: Andrea Scozzari

Received: 2 December 2021

Accepted: 13 January 2022

Published: 19 January 2022

Publisher's Note: MDPI stays neutral with regard to jurisdictional claims in published maps and institutional affiliations.

Copyright: (C) 2022 by the authors. Licensee MDPI, Basel, Switzerland. This article is an open access article distributed under the terms and conditions of the Creative Commons Attribution (CC BY) license (https:// creativecommons.org/licenses/by/ $4.0 /)$.

\begin{abstract}
Graphs serve as one of the main tools for the mathematical modeling of various human problems. Fuzzy graphs have the ability to solve uncertain and ambiguous problems. The cubic graph, which has recently gained a position in the fuzzy graph family, has shown good capabilities when faced with problems that cannot be expressed by fuzzy graphs and interval-valued fuzzy graphs. Simultaneous application of fuzzy and interval-valued fuzzy membership indicates a high flexibility in modeling uncertainty issues. The vertex cover is a fundamental issue in graph theory that has wide application in the real world. The previous definition limitations in the vertex covering of fuzzy graphs has directed us to offer new classifications in terms of cubic graph. In this study, we introduced the strong vertex covering and independent vertex covering in a cubic graph with strong edges and described some of its properties. One of the motives of this research was to examine the changes in the strong vertex covering number of a cubic graph if one vertex is omitted. This issue can play a decisive role in covering the graph vertices. Since many of the problems ahead are of hybrid type, by reviewing some operations on the cubic graph we were able to determine the strong vertex covering number on the most important cubic product operations. Finally, two applications of strong vertex covering and strong vertex independence are presented.
\end{abstract}

Keywords: cubic graph; cubic strong edge; strong vertex covering number; strong vertex independent set

\section{Introduction}

Graphs have long been used to describe objects and the relationships among them. The vertex cover (VC) of a graph $\mathrm{G}$ is an arrangement of vertices, in which every edge in $\mathrm{G}$ has at least one end point in this set provided that each vertex in $\mathrm{G}$ is at least adjacent to one edge. The VC number, also called the minimum VC number, is the minimum cardinality of a set of vertices that covers all edges in G. Determining the set of VCs with the least cardinality for a given graph is a classic optimization problem in computer science and an NP-hard problem in which it is not possible to design an algorithm that guarantees the optimal answer in polynomial time. In fact, the VC problem was one of Karp's 21 NP-complete problems, and is, therefore, a classical NP-complete problem in complexity theory.

Considering the VC issue, Hastad [1] confirmed that approximating VC within constant factors less than 7/6 serves as the NP-hard. The Hastad factor to 1.36 was progressed by Dinur and Safra [2]. The problems of randomness and the probability theory of minimum weight edge covering problem are mentioned by $\mathrm{Ni}$ [3].

Fuzzy theory is one of the best and most powerful tools for modeling problems in examining the relationships among uncertainties in the real world. This concept gained popularity with the introduction of the fuzzy set by Zadeh [4], and fuzzy graph (FG) by Rosenfeld [5], as they are characterized by two membership functions in $[0,1]$ for vertices and edges of a graph. Bhutani and Rosenfeld [6] introduced the concept of strong edges. 
Bhattacharya [7] presented some observations on FGs and some operations on FGs were introduced by Mordeson and Peng [8]. Akram and Dudek [9] presented the idea of an interval-valued fuzzy graph (IVFG) in 2011. Atanassov [10] adopted the idea of an element membership and non-membership in a set and proposed the idea of intuitionistic fuzzy sets. Rashmanlou et al. [11] studied categorical properties of an intuitionistic fuzzy graph (IFG). Kosari et al. [12-17] conducted research on graphs and vague graphs. Some concepts of IFG were studied by Shao et al. [18]. Talebi [19] introduced Cayley fuzzy graphs to the fuzzy group. Talebi et al. $[20,21]$ introduced some new concepts of the interval-valued intuitionistic fuzzy graph (IVIFG). Some researchers have studied the concepts of graph structure [22-26].

The concept of covering in FG was introduced by Somasundaram [27] who defined VC and edge covering in FGs using effective edges and scalar cardinality. According to this definition, a VC in an FG G is a subset D of vertices so that for each effective arc there is at least one of the two end points in D. Somasundaram defined the notion of coverings in a smaller domain of effective edges using scalar cardinality. According to this definition, for any FG without effective edges, the VC is an empty set. Please note that each effective edge is strong, but a strong edge need not be effective. The credibility theory to find the minimum fuzzy weight edge cover in an FG was discussed by $\mathrm{Ni}$ [28] in 2008. Manjusha and Sunitha [29] introduced covering, matching, and paired domination in FGs using strong edges. Sahoo et al. [30] studied covering and paired domination in IFGs. Vinothkumar and Ramya [31] introduced covering in operations on FGs. Senthilkumar and Ponnappan [32] analyzed the idea of strong support VC of FG using strong arc.

Jun et al. [33] introduced the idea of the cubic set (CS) in the form of a combination of FS and IVFS, serving as a more general tool for modeling uncertainty and ambiguity. Through applying this concept, we can solve various problems instigated by uncertainties and have the best choice using CSs in decision-making. Jun et al. [34] combined neutrosophic sets with CSs and proposed the neutrosophic CS idea, and defined different operations. Aided by his colleagues, he also applied this concept to algebraic structures [35-38]. Kang and Kim [39] investigated CSs mappings. Muhiuddin et al. [40] presented the stable CSs idea. Rashid et al. [41] introduced the concept of a cubic graph (CG) where they introduced many new types of graphs and their applications. Muhiuddin et al. [42] provided a modified definition of a CG. Kishore Kumar et al. [43] examined the regularity concept in $\mathrm{CG}$.

The VC is one of the graph-theory concepts that has various applications such as the installation of road cameras to control traffic, dynamic detection of competition conditions in parallel programming, and finding phylogenetic trees based on protein domain information. Additionally, one of the applications of the minimum VC set is in scheduling issues. A timing problem can be modeled as a graph, where vertices represent tasks or times, and the edge between vertices means there is a conflict between those times or tasks. In fact, finding the minimum number of tasks that must be eliminated to resolve all conflicts is equivalent to finding the minimum VC. The minimum VC problem can also be used to model many real-world situations in the fields of circuit design, telecommunications, network flow, and so on.

In this research, aiming at expanding the concept of covering, and we studied the VC in a CG. We introduced the VC considering cubic strong edges (CSE). We introduced the strong VC and independent VC in a CG with CSEs and described some of its properties. The status of the CG coverage, if one vertex is removed, has been one of the objectives of this research. In this regard, we were able to compare the strong vertex covering set (SVCS) number and strong vertex independent set (SVIS) number in a CG before and after removing a vertex. In the following, since many of the surrounding problems are a combination of different topics, we determined the SCVS number on the most important fuzzy operations in the CG. Finally, two applications of SVCS and SVIS are presented. 
Although the CG in graph theory is also referred to as 3-regular graphs, the meaning of the CG throughout this article means a cubic fuzzy graph consisting of an IVFG and an FG.

\section{Preliminaries}

In this section, some basic concepts of graphs and fuzzy graphs are outlined to enter the main discussion.

A graph consists of a pair $G=(V, E)$, where $V$ is the set of vertices and $E$ is the set of edges of $G$. $H=\left(V^{\prime}, E^{\prime}\right)$ is known as a sub graph of a graph $G$ whenever $V^{\prime} \subseteq V$ and $E^{\prime} \subseteq E$. A vertex and an incident edge are called to cover each other in $G$. A VC in $G$ is a set of vertices that cover all edges of $G$. The minimum number of vertices in a VC of $G$ is the VC number $\alpha_{0}(G)$ of $G$. A set of vertices in $G$ is independent if no two vertices in the set are linked. The vertex independence number $\beta_{0}(G)$ of $G$ is the maximum cardinality of an independent set of vertices in $G$.

A fuzzy set $A \subseteq V$ is described by its membership function of $\sigma_{A}: V \rightarrow[0,1]$, where $\sigma_{A}(x)$ means the degree to which $x$ belongs to $A$. For notational convenience, we write $A(x)$ instead of $\sigma_{A}(x)$. We use the symbol $F(V)$ to denote the set of all fuzzy subsets of $V$.

If $\sigma_{1}, \sigma_{2} \in F(V)$, then we define

$$
\begin{aligned}
& \sigma_{1}(x) \wedge \sigma_{2}(x)=\min \left\{\sigma_{1}(x), \sigma_{2}(x)\right\}, \\
& \sigma_{1}(x) \vee \sigma_{2}(x)=\max \left\{\sigma_{1}(x), \sigma_{2}(x)\right\} \\
& \sigma_{1} \subseteq \sigma_{2} \Leftrightarrow \sigma_{1}(x) \leq \sigma_{2}(x), \forall x \in V .
\end{aligned}
$$

An interval-valued number means a closed subinterval $[s, t]$ of $[0,1]$, so that $0 \leq s \leq$ $t \leq 1$. The set of interval-valued numbers is denoted by $D[0,1]$. For two interval-valued numbers $c_{1}=\left[s_{1}, t_{1}\right]$ and $c_{2}=\left[s_{2}, t_{2}\right]$, we define

$$
\begin{aligned}
& r \min \left\{c_{1}, c_{2}\right\}=\left[\min \left\{s_{1}, s_{2}\right\}, \min \left\{t_{1}, t_{2}\right\}\right], \\
& c_{1} \geq c_{2} \Leftrightarrow s_{1} \geq s_{2}, t_{1} \geq t_{2} .
\end{aligned}
$$

A function $\mu: V \rightarrow D[0,1]$ is said to be an interval-valued fuzzy set in $V$. For $x \in V$, $\left[\mu^{L}(x), \mu^{U}(x)\right]$ is considered to be the membership degree of $x$, where $\mu^{L}, \mu^{U}: V \rightarrow[0,1]$. For every $\mu, v \in D[0,1]$, we define

$$
\mu \subseteq \nu \Leftrightarrow \mu(x) \leq \nu(x), \quad \forall x \in V
$$

Additionally, we have

$$
\begin{aligned}
& \bigcup\left[\mu_{i}^{L}(x), \mu_{i}^{U}(x)\right]=\left[\sup \mu_{i}^{L}(x), \sup \mu_{i}^{U}(x)\right], \\
& \bigcap\left[\mu_{i}^{L}(x), \mu_{i}^{U}(x)\right]=\left[\inf \mu_{i}^{L}(x), \inf \mu_{i}^{U}(x)\right],
\end{aligned}
$$

for all $x \in V, i=1,2, \ldots, n$.

The FG $G=(\sigma, \rho)$ over $V$ is a non-empty set $V$ together with a pair of functions $\sigma: V \rightarrow[0,1]$ and $\rho: V \times V \rightarrow[0,1]$, so that for all $x, y \in V, \rho(x y) \leq \sigma(x) \wedge \sigma(y)$ where $\sigma(x)$ and $\rho(x y)$ represent the membership values of the vertex $x$ and the edge $x y$ in $G$, respectively. The FG $H=(\varphi, \psi)$ is named the partial fuzzy subgraph of $G=(\sigma, \rho)$ if $\varphi \subseteq \sigma$ and $\psi \subseteq \rho$. Two vertices of $x$ and $y$ in the FG $G$ are named the adjacent neighbors if $\rho(x y)>0$. The set of all neighbors of $x$ is shown by $N(x)$. The FG $G=(\sigma, \rho)$ is named the complete FG if $\rho(x y)=\sigma(x) \wedge \sigma(y)$, for all $x, y \in V$.

The order and size of the FG $G=(\sigma, \rho)$ are described as $p=\sum_{x \in V} \sigma(x)$, $q=\sum_{x y \in V \times V} \rho(x y)$. Additionally, the scalar cardinality of $S \subseteq V$ is described as $|S|=$ $\sum_{x \in S} \sigma(x)$.

A path $P$ of length $n$ is a sequence of distinct vertices $x_{0}, x_{1}, \ldots, x_{n}$, so that $\rho\left(x_{i-1}, x_{i}\right)>$ $0, i=1,2, \ldots, n$, and the membership degree of a weakest edge is defined as its strength. 
The strength of connectedness between two vertices $x$ and $y$ is defined as the maximum of the strength of a path between $x$ and $y$, denoted by $\operatorname{CONN}(x, y)$.

An edge of an FG $G=(\sigma, \rho)$ is named strong if its weight is at least as great as the strength of connectedness of its end vertices when it is deleted. An FG $G$ is called the strong $F G$ if each edge in $G$ is a strong edge. The node $y$ is named strong neighbor of $x$ if the edge $x y$ is strong. The set of all strong neighbors of $x$ is named the strong neighbor of $x$ and is shown by $N_{S}(x)$. The closed strong neighbor is described as $N_{S}[x]=N_{S}(x) \cup\{x\}$.

Definition 1 ([33]). A CS in $V$ is defined as follows

$$
A=\left\{\left\langle\left[\mu^{L}(x), \mu^{U}(x)\right], \sigma(x)\right\rangle \mid x \in V\right\},
$$

where $\left[\mu^{L}(x), \mu^{U}(x)\right]$ is an interval-valued fuzzy membership degree and $\sigma(x)$ is a fuzzy membership degree of $x$ in $A$.

$A C S A$ in $V$ is named to be an internal CS if $\mu^{L}(x) \leq \sigma(x) \leq \mu^{U}(x)$, and said to be an external CS if $\sigma(x) \notin\left[\mu^{L}(x), \mu^{U}(x)\right]$, for all $x \in V$.

Definition 2 ([42]). A CG over $V^{*} \neq \varnothing$ is a pair $\mathcal{G}=(A, B)$ where $A$ is a CS in $V^{*}$ and $B$ is a CS in $V^{*} \times V^{*}$, so that for all $x y \in V^{*} \times V^{*}$

$$
\begin{aligned}
& \mu_{B}^{L}(x y) \leq \mu_{A}^{L}(x) \wedge \mu_{A}^{L}(y), \\
& \mu_{B}^{U}(x y) \leq \mu_{A}^{U}(x) \wedge \mu_{A}^{U}(y), \\
& \sigma_{B}(x y) \leq \sigma_{A}(x) \wedge \sigma_{A}(y) .
\end{aligned}
$$

The underlying graph of a $C G \mathcal{G}$ is $\mathcal{G}^{*}=\left(V^{*}, E^{*}\right)$, where

$$
\begin{aligned}
& V^{*}=\left\{x \mid\left[\mu_{A}^{L}(x), \mu_{A}^{U}(x)\right]>0, \sigma_{A}(x)>0\right\}, \\
& E^{*}=\left\{x y \mid\left[\mu_{B}^{L}(x y), \mu_{B}^{U}(x y)\right]>0, \sigma_{B}(x y)>0\right\}, \forall x, y \in V^{*} .
\end{aligned}
$$

Definition 3 ([42]). A CG $\mathcal{G}$ on $V^{*}$ is known as a complete $C G$ if for all $x y \in E^{*}$,

$$
\begin{aligned}
& \mu_{B}^{L}(x y)=\mu_{A}^{L}(x) \wedge \mu_{A}^{L}(y), \\
& \mu_{B}^{U}(x y)=\mu_{A}^{U}(x) \wedge \mu_{A}^{U}(y), \\
& \sigma_{B}(x y)=\sigma_{A}(x) \wedge \sigma_{A}(y) .
\end{aligned}
$$

Definition 4 ([42]). Let $\mathcal{G}=(A, B)$ be a $C G$ on $V^{*}$. A cubic path in $\mathcal{G}$ is a sequence $P_{c}$ : $x_{1}, x_{2}, \ldots, x_{r}$ of distinct vertices of $V$, so that $\left[\mu_{B}^{L}\left(x_{i-1}, x_{i}\right), \mu_{B}^{U}\left(x_{i-1}, x_{i}\right)\right]>0$ and $\sigma_{B}\left(x_{i-1}, x_{i}\right)>$ 0 , for $i=1,2, \ldots, r$. The strength of $P_{c}$ is defined as

$$
S\left(P_{c}\right)=\left\langle\bigcap_{i=1}^{r}\left[\mu_{B}^{L}\left(x_{i-1}, x_{i}\right), \mu_{B}^{U}\left(x_{i-1}, x_{i}\right)\right], \bigwedge_{i=1}^{r} \sigma_{B}\left(x_{i-1}, x_{i}\right)\right\rangle .
$$

The strength of connectedness between $x$ and $y$ is shown by $\left\langle\left[\mu_{B}^{\infty L}(x y), \mu_{B}^{\infty U}(x y)\right], \sigma_{B}^{\infty}(x y)\right\rangle$ and it is the maximum of the strengths of all cubic paths between $x, y$.

Definition 5. An edge $x y$ in $C G \mathcal{G}$ is named cubic strong edge (CSE) if

$$
\left[\mu_{B}^{L}(x y), \mu_{B}^{U}(x y)\right] \geq\left[\mu_{B}^{\infty L}(x y), \mu_{B}^{\infty U}(x y)\right], \quad \sigma_{B}(x y) \geq \sigma_{B}^{\infty}(x y) .
$$


Definition 6. The vertex cardinality and edge cardinality of a $C G \mathcal{G}=(A, B)$ are described as

$$
\begin{aligned}
& p=\sum_{x \in V^{*}}\left(\frac{1-\mu_{A}^{L}(x)+\mu_{A}^{U}(x)+\sigma_{A}(x)}{3}\right), \\
& q=\sum_{x y \in E^{*}}\left(\frac{1-\mu_{B}^{L}(x y)+\mu_{B}^{U}(x y)+\sigma_{B}(x y)}{3}\right) .
\end{aligned}
$$

Definition 7. The cardinality of $S \subseteq V^{*}$ in $C G \mathcal{G}$ is described as

$$
|S|=\sum_{x \in S}\left(\frac{1-\mu_{A}^{L}(x)+\mu_{A}^{U}(x)+\sigma_{A}(x)}{3}\right) .
$$

Definition 8. Let $\mathcal{G}$ be a CG. The strong neighbor of $x$ is described as

$$
N(x)=\left\{y \in V^{*} \mid x y \text { be a CSE }\right\} \text {. }
$$

The strong neighborhood degree of $x$ is defined as

$$
D N(x)=\left\langle\left[\sum_{w \in N(x)} \mu^{L}(w), \sum_{w \in N(x)} \mu^{U}(w)\right], \sum_{w \in N(x)} \sigma(w)\right\rangle .
$$

The minimum and maximum cardinality of the strong neighborhood of $\mathcal{G}$ are shown by $\delta_{N}$ and $\Delta_{N}$, respectively.

Definition 9. In a CG $\mathcal{G}$, a vertex $x \in V^{*}$ is named an isolated vertex if for any $y \in V^{*}$ where $x \neq y, x y$ is not a cubic edge.

Definition 10. The degree of a vertex $x$ in a CG is represented as

$$
d(x)=\sum_{x \neq y} \frac{1-\mu_{B}^{L}(x y)+\mu_{B}^{U}(x y)+\sigma_{B}(x y)}{3} .
$$

The minimum and maximum degree of a vertex of $\mathcal{G}$ are shown by $\delta(\mathcal{G})$ and $\Delta(\mathcal{G})$, respectively.

The main abbreviations used in this article are given in Table 1.

Table 1. Abbreviations.

FG
VC
CS
CG
CSE
SVCS
SVIS

FG

$\mathrm{VC}$

CG

CSE

SVIS
Fuzzy graph Vertex covering

Cubic set

Cubic graph Cubic strong edge

Strong vertex covering set Strong vertex independent set

\section{Vertex Covering and Independent Covering in Cubic Graphs}

In this section, the vertex covering and independent covering are discussed in cubic graphs and some of their properties are compared.

Definition 11. Let $\mathcal{G}=(A, B)$ be a CG. A vertex and a CSE adjacent to it are named strong cover for each other. A strong vertex covering set (SVCS) in $\mathcal{G}$ is the set $C$ of vertices so that each CSE in $\mathcal{G}$ is adjacent with at least one vertex in $C$. 
The subset $C$ is called the minimal SVCS of the CG $\mathcal{G}$ whenever $C \backslash\{x\}$ is not an SVCS, for all $x \in C$.

The minimum cardinality among all the minimal SVCSS of $\mathcal{G}$ is named the SVCS number of $\mathcal{G}$ and it is shown by $\alpha_{s}(\mathcal{G})$ or simply $\alpha_{s}$.

An SVCS with minimum cardinality in CG $\mathcal{G}$ is named the minimum SVCS and it is denoted as $\alpha_{s}-$ set.

Remark 1. Every SVCS is a VC set, but a VC set need not be an SVCS.

The above definition is supported by the following example.

Example 1. Consider the CG $\mathcal{G}$ as drawn in Figure 1. The CSEs are $a b, c d$, and ef. The minimal SVCSs in Figure 1 are as follows:

$$
\begin{array}{llll}
C_{1}=\{a, c, e\}, & C_{2}=\{a, d, e\}, & C_{3}=\{a, c, f\}, & C_{4}=\{a, d, f\}, \\
C_{5}=\{b, d, f\}, & C_{6}=\{b, c, f\}, & C_{7}=\{b, d, e\}, & C_{8}=\{b, c, e\} .
\end{array}
$$

From the cardinality calculation of the above SVCSs, we have

$$
\begin{array}{llll}
\left|C_{1}\right|=1.59, & \left|C_{2}\right|=1.75, & \left|C_{3}\right|=1.46, & \left|C_{4}\right|=1.62, \\
\left|C_{5}\right|=1.72, & \left|C_{6}\right|=1.56, & \left|C_{7}\right|=1.85, & \left|C_{8}\right|=1.69 .
\end{array}
$$

It is clear that $C_{3}$ has the minimum cardinality among other SVCSs. Therefore, $\alpha_{\mathcal{S}}(\mathcal{G})=1.46$ and $C_{3}$ is the $\alpha_{s}$-set of $\mathcal{G}$.

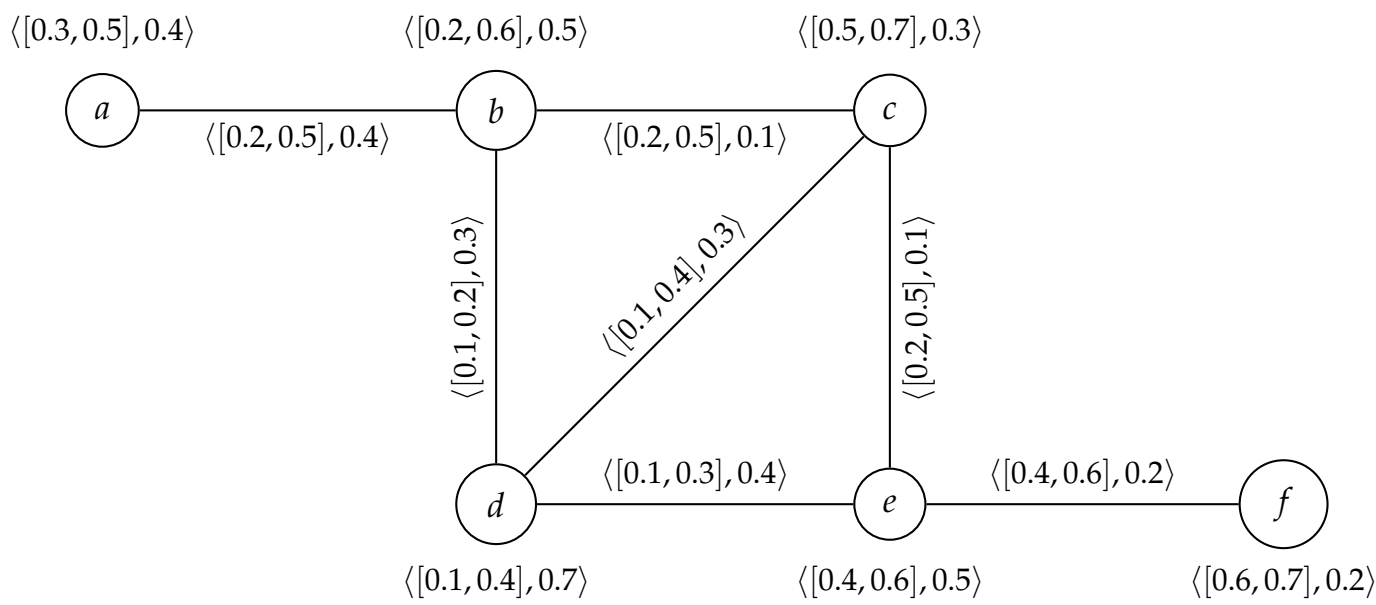

Figure 1. The CG $\mathcal{G}$.

Proposition 1. If $\mathcal{G}$ is a complete $C G$, then, $\alpha_{\mathcal{S}}(\mathcal{G})=p-t$, where $t$ is the maximum cardinality of a vertex in $\mathcal{G}$.

Proof. Since $\mathcal{G}$ is a complete CG, then, all edges are CSE, and each vertex is adjacent to all other vertices. Hence, each set with $(n-1)$ vertices forms an SVCS of $\mathcal{G}$. Let $x$ be a vertex with the maximum cardinality of $t$ in $\mathcal{G}$. By removing this vertex from the vertex set, the remaining vertices will form an SVCS with the minimum of cardinality. Then, $\alpha_{s}(\mathcal{G})=p-t$.

Proposition 2. For a complete bipartite $C G \mathcal{G}$ with partite sets of $A_{1}$ and $A_{2}$,

$$
\alpha_{s}(\mathcal{G})=\left|A_{1}\right| \wedge\left|A_{2}\right|
$$


Proof. In complete bipartite $\mathrm{CG} \mathcal{G}=(A, B)$, all edges are CSE. Additionally, each vertex in $A_{1}$ is adjacent to all vertices in $A_{2}$ and vice-versa. Since each CSE has one end in $A_{1}$ and the other end in $A_{2}$, the SVCSs in $\mathcal{G}$ are $A_{1}, A_{2}$ and $A_{1} \cup A_{2}$. Hence, $\alpha_{s}(\mathcal{G})=\left|A_{1}\right| \wedge\left|A_{2}\right|$.

Proposition 3. Let $\mathcal{G}$ be a CG so that $\mathcal{G}^{*}$ is a cycle. Then, $\alpha_{s}$ is the minimum cardinality of SVCSs C so that $|C| \geq\left\lceil\frac{n}{2}\right\rceil$.

Proof. Let $\mathcal{G}$ be a cubic cycle. Then, every edge is CSE. On the other hand, the number of vertices in an SVCS of $\mathcal{G}$ and $\mathcal{G}^{*}$ is the same, because each edge in both graphs is strong. Since the vertex covering number of $\mathcal{G}^{*}$ is $\left\lceil\frac{n}{2}\right\rceil$, then the minimum number of vertices in an SVCS of $\mathcal{G}$ is $\left\lceil\frac{n}{2}\right\rceil$. Hence, the result follows.

In the following theorems, we compare the SVCS number if one vertex is omitted.

Theorem 1. If $\mathcal{G}$ is a $C G$ and $x \in V^{*}$, then, $\alpha_{s}(\mathcal{G}-x) \leq \alpha_{s}(\mathcal{G})$.

Proof. Let $C$ be a minimum SVCS of $\mathcal{G}$.

Case i. Suppose $x \notin C$.

Consider $u$ and $v$ as two adjacent vertices of $\mathcal{G}-x$. Then, they are also adjacent in $\mathcal{G}$. Since $C$ is an SVCS, then, $u \in C$ or $v \in C$. Therefore, $C$ is an SVCS of $\mathcal{G}-x$. Hence, $\alpha_{s}(\mathcal{G}-x) \leq \alpha_{s}(\mathcal{G})$.

Case ii. Suppose $x \in C$.

Consider the set $C_{1}=C \backslash\{x\}$. Assume $u$ and $v$ as two nodes of $\mathcal{G}-x$ which are adjacent in $\mathcal{G}-x$. According to the above argument, $u \in C$ or $v \in C$.

Since $u \neq x$ and $v \neq x, u \in C_{1}$ or $v \in C_{1}$. Thus, $C_{1}$ is an SVCS of $\mathcal{G}-x$. Therefore,

$$
\alpha_{s}(\mathcal{G}-x) \leq\left|C_{1}\right|<|C|=\alpha_{s}(\mathcal{G}) .
$$

Hence, from both cases $\alpha_{s}(\mathcal{G}-x) \leq \alpha_{s}(\mathcal{G})$.

Theorem 2. $\alpha_{s}(\mathcal{G}-x)<\alpha_{s}(\mathcal{G})$ if and only if there is a $\alpha_{s}$-set $C$, so that $x \in C$.

Proof. Suppose $C$ is a $\alpha_{s}$-set of $\mathcal{G}$, so that $x \in C$. Let $C_{1}=C \backslash\{x\}$. According to Theorem 1 , $C_{1}$ is an SVCS of $\mathcal{G}-x$. Thus,

$$
\alpha_{s}(\mathcal{G}-x) \leq\left|C_{1}\right|<|C|=\alpha_{s}(\mathcal{G}) .
$$

Hence, $\alpha_{s}(\mathcal{G}-x)<\alpha_{s}(\mathcal{G})$.

Conversely, suppose $\alpha_{\mathcal{S}}(\mathcal{G}-x)<\alpha_{\mathcal{S}}(\mathcal{G})$. Let $C_{1}$ be a minimum vertex covering set of $\mathcal{G}-x$. Then, $C_{1}$ cannot be an SVCS of $\mathcal{G}$. (Because otherwise $\alpha_{s}(\mathcal{G}) \leq\left|C_{1}\right|=\alpha_{s}(\mathcal{G}-x)$ ).

Let $C=C_{1} \cup\{x\}$, then, $C$ is an SVCS of $\mathcal{G}$. Since $\alpha_{s}(\mathcal{G}-x)<\alpha_{s}(\mathcal{G}), C$ is an SVCS of $\mathcal{G}$ and it also contains a vertex $x$.

Corollary 1. $\alpha_{s}(\mathcal{G}-x)=\alpha_{s}(\mathcal{G})$ if and only if $x$ does not belong to any minimum SVCS of $\mathcal{G}$.

Theorem 3. If $\mathcal{G}=(A, B)$ is a $C G$ without isolated vertex, then, $\alpha_{s}(\mathcal{G}) \leq \frac{p}{2}$.

Proof. Let $\mathcal{G}=(A, B)$ be a CG without isolated vertex and the set $C$ be an SVCS of $\mathcal{G}$. Then, $V^{*} \backslash C$ is also an SVCS of $\mathcal{G}$. Because $\mathcal{G}$ does not have an isolated vertex, thus,

$$
\begin{aligned}
\alpha_{S}(\mathcal{G}) & =\min \left\{|C|,\left|V^{*} \backslash C\right|\right\}, \\
\alpha_{S}(\mathcal{G}) & \leq \frac{p}{2} .
\end{aligned}
$$

Corollary 2. In a $C G \mathcal{G}, \alpha_{s}(\mathcal{G}) \geq \delta(\mathcal{G})$. 
Proof. Assume $\mathcal{G}$ to be a CG and $C$ is an SVCS of $\mathcal{G}$. Then, $|C| \geq d(x) \geq \delta(\mathcal{G})$, for all $x \in V^{*}$. Hence, $\alpha_{s}(\mathcal{G}) \geq \delta(\mathcal{G})$.

Remark 2. The above results show that it should be $\delta(\mathcal{G}) \leq \alpha_{s}(\mathcal{G}) \leq \frac{p}{2}$.

In the following definition, we introduce the strong vertex independent set in a cubic graph.

Definition 12. Let $\mathcal{G}$ be a $C G$. Two vertices of $\mathcal{G}$ are named strongly independent if there is no CSE between them. A cubic subset $F \subseteq V^{*}$ is known as the strong vertex independent set (SVIS) of $\mathcal{G}$ if each two vertices of $F$ are strongly independent. An SVIS is called maximal SVIS if no superset of $F$ is an SVIS. The maximum cardinality of SVISs in $\mathcal{G}$ is named the SVIS number and it is denoted by $\beta_{s}(\mathcal{G})$ or simply by $\beta_{s}$.

Example 2. In Figure 1, only $C_{7}$ is a $\beta_{s}$-set of $\mathcal{G}$ with the maximum cardinality, so $\beta_{s}(\mathcal{G})=$ $\left|C_{7}\right|=1.85$.

Proposition 4. If $\mathcal{G}$ is a complete $C G$, then, $\beta_{s}(\mathcal{G})=u$, where $u$ is the maximum cardinality of a vertex in $\mathcal{G}$.

Proof. Since $\mathcal{G}$ is a complete CG, then, all edges are CSE, and each vertex is adjacent to all other vertices. Hence, $F=\{x\}$ is the only SVIS in $\mathcal{G}$ for each $x \in V^{*}$. Thus, $\beta_{s}(\mathcal{G})=u$ where $u$ is the maximum cardinality of a vertex in $\mathcal{G}$.

Proposition 5. For a complete bipartite $C G \mathcal{G}=(A, B)$ with partite sets of $A_{1}$ and $A_{2}$, we have

$$
\beta_{s}(\mathcal{G})=\left|A_{1}\right| \vee\left|A_{2}\right| \text {. }
$$

Proof. In complete bipartite $\mathrm{CG} \mathcal{G}=(A, B)$, all edges are CSE. Additionally, each vertex in $A_{1}$ is adjacent to all vertices in $A_{2}$ and vice-versa. Hence, the SVISs in $\mathcal{G}$ are $A_{1}$ and $A_{2}$. Then, $\beta_{S}(\mathcal{G})=\left|A_{1}\right| \vee\left|A_{2}\right|$.

Proposition 6. Let $\mathcal{G}$ be a $C G$, so that $\mathcal{G}^{*}$ is a cycle. Then, $\beta_{s}(\mathcal{G})$ is the maximum cardinality of SVISs F, so that $|F| \leq\left\lfloor\frac{n}{2}\right\rfloor$.

Proof. Since $\mathcal{G}$ is a cubic cycle, then, every edge is CSE. Furthermore, the number of nodes in an SVIS of $\mathcal{G}$ and $\mathcal{G}^{*}$ are the same, because each edge in both graphs is strong. Since that strong independence number of $\mathcal{G}^{*}$ is $\left\lfloor\frac{n}{2}\right\rfloor$, then, the maximum number of vertices in an SVIS of $\mathcal{G}$ is $\left\lfloor\frac{n}{2}\right\rfloor$. Thus, the results will be obtained.

Theorem 4. Let $\mathcal{G}$ be a $C G$ so that $\mathcal{G}^{*}$ is a cycle with $V^{*}\left(\mathcal{G}^{*}\right)=\{0,1,2, \ldots, n-1\}, E^{*}\left(\mathcal{G}^{*}\right)=$ $\{(i, j) \mid i-j \equiv 1$ (mode $n)\}$, and $n$ is an even number. If $|2 i| \geq|2 j-1|$, for any $0 \leq i \leq \frac{n}{2}$, $1 \leq j \leq \frac{n}{2}$, then, $\beta_{s}(\mathcal{G})=|\{0,2,4, \ldots, n-2\}|$.

Proof. It is clear that the set $S=\{0,2, \ldots, n-2\}$ is a maximal SVIS of $\mathcal{G}$. We prove that for any maximal SVIS $D$ of $\mathcal{G},|D| \leq|S|$.

If $D \cap S=\varnothing$, then, $D=\{1,3, \ldots, n-1\}$. Thus,

$$
|D|=\sum_{j=1}^{n / 2}|2 j-1| \leq \sum_{j=0}^{\frac{n-2}{2}}|2 j|=|S| .
$$

Based on the hypothesis, for any subset $X$ of $\{1,3, \ldots, n-1\}$, if $Y$ is a subset of $\{0,2, \ldots, n-2\}$ so that the cardinal of $X$ is less than or equal to the cardinal of $Y$, then, $|X| \leq|Y|$. 
Now, $D \cap S \neq \varnothing$, using $D=(D \cap S) \cup(D \backslash S)$, we have

$$
|D|=|D \cap S|+|D \backslash S| \leq|D \cap S|+|\{0,2, \ldots, n-2\} \backslash(D \cap S)|=|\{0,2, \ldots, n-2\}| .
$$

Example 3. Consider the cubic cycle $\mathcal{G}$ as depicted in Figure 2. The SVCSs in Figure 2 are as follows.

$$
\begin{array}{lll}
C_{1}=\{a, c, e\}, & C_{2}=\{b, d, f\}, & C_{3}=\{a, b, d, e\}, \\
C_{4}=\{a, b, c, e\}, & C_{5}=\{a, c, d, f\}, & C_{6}=\{a, c, d, e\}, \\
C_{7}=\{a, b, c, d, e\}, & C_{8}=\{b, c, d, e, f\}, & C_{9}=\{a, c, d, e, f\}, \\
C_{10}=\{a, b, c, d, e, f\} . & &
\end{array}
$$

After calculations, $\alpha_{s}(G)=1.49$ and $C_{2}$ is $\alpha_{s}$-set. Additionally, the SVISs are as below

$$
F_{1}=\{a, e, c\}, \quad F_{2}=\{b, d, f\} .
$$

Therefore, $\beta_{s}(G)=1.59$, and $F_{1}$ is $\beta_{s}$-set.

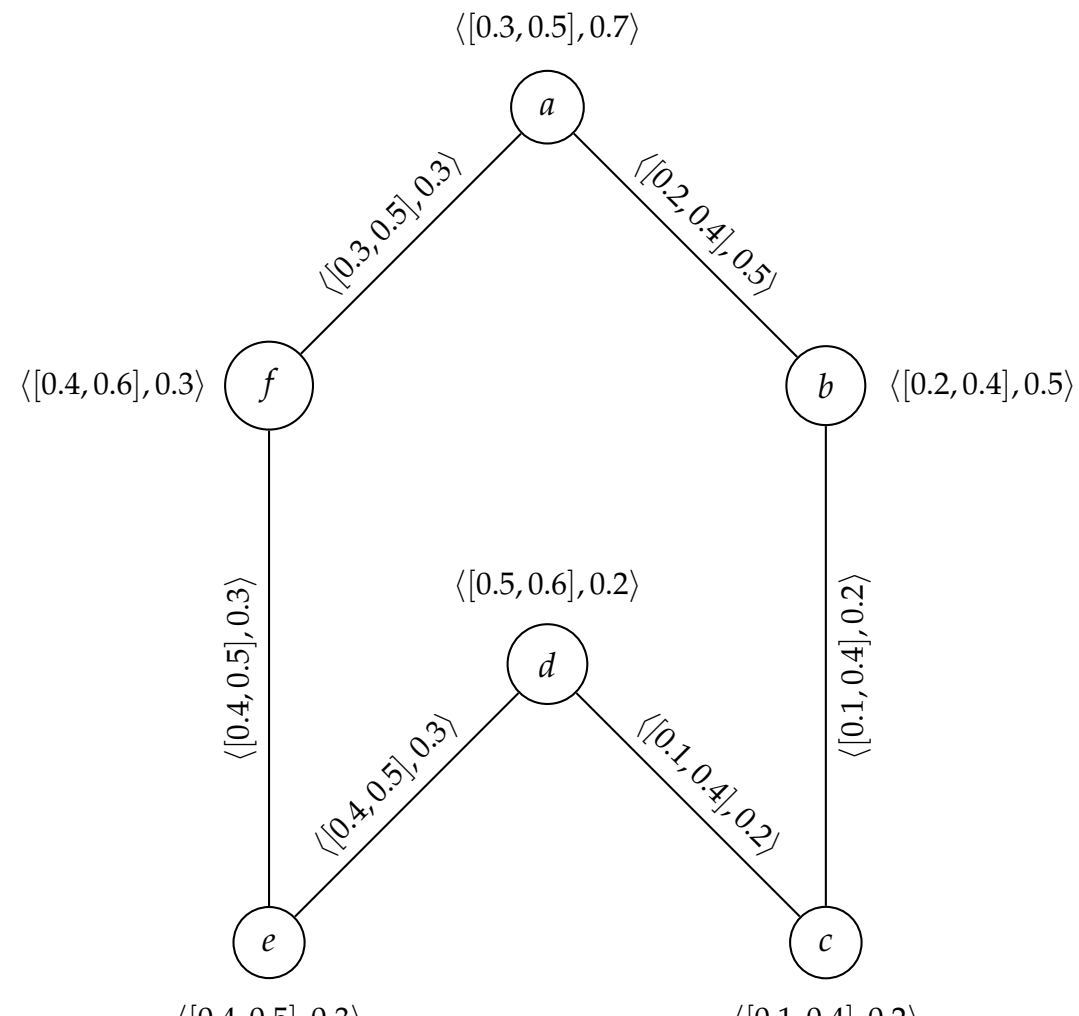

$\langle[0.4,0.5], 0.3\rangle$

$\langle[0.1,0.4], 0.2\rangle$

Figure 2. Cubic cycle $\mathcal{G}$.

Proposition 7. Let $\mathcal{G}$ be a $C G$ over $\mathcal{G}^{*}$. If $x$ is a pendant vertex in $\mathcal{G}^{*}$, then there is a $\beta_{s}$-set $F$ so that $x \in F$.

Proof. Let $x$ be a pendant vertex in $\mathcal{G}^{*}, y$ is its neighbor, and $F$ is a $\beta_{s}$-set in $\mathcal{G}$. Then, $x \notin F$ if and only if $y \in F$. If $x \notin F$, let $F^{\prime}=(F \cup\{x\}) \backslash\{y\}$, then, $\left|F^{\prime}\right|=|F|$. Thus, $F^{\prime}$ is a $\beta_{s}$-set.

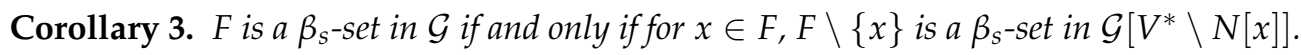


The following theorem examines and compares the strong vertex independent number in a cubic graph if one vertex is omitted.

Theorem 5. Let $\mathcal{G}$ be a $C G$ and $x \in V^{*}$. Then, $\beta_{\mathcal{S}}(\mathcal{G}-x)<\beta_{\mathcal{S}}(\mathcal{G})$ if and only if there is a maximum SVIS F of $\mathcal{G}-x$, so that $N(x) \cap F=\varnothing$.

Proof. Suppose $\beta_{S}(\mathcal{G}-x)<\beta_{S}(\mathcal{G})$. Consider $F$ to be a $\beta_{s}$-set of $\mathcal{G}$. If $x \in F$, then $F \backslash\{x\}$ is a $\beta_{s}$-set of $\mathcal{G}-x$. Since $x$ is not adjacent to any vertex in $\mathcal{G}$, then $N(x) \cap F=\varnothing$.

Consider $x \notin F$ for any maximum SVIS $F$ of $\mathcal{G}$. Then, for any set $F, F$ is an SVIS in $\mathcal{G}-x$, which implies that $\beta_{S}(\mathcal{G}-x) \geq \beta_{S}(\mathcal{G})$. This is a contradiction.

Conversely, suppose $F$ to be an SVIS of $\mathcal{G}-x$, so $N(x) \cap F=\varnothing$. We claim that $F$ is also an SVIS in $\mathcal{G}$. Consider $u, v \in F$ which are adjacent in $\mathcal{G}$. Then, $u \neq x$ and $v \neq x$. Since $N(x) \cap F=\varnothing, u$ and $v$ are adjacent in $\mathcal{G}-x$ which contradicts the SVIS of $F$ in $\mathcal{G}-x$.

Let $F_{1}=F \cup\{x\}$. Since $N(x) \cap F=\varnothing, F_{1}$ is an SVIS in $\mathcal{G}$. Thus,

$$
\beta_{S}(\mathcal{G}) \geq\left|F_{1}\right|>|F|=\beta_{S}(\mathcal{G}-x), \quad \Rightarrow \quad \beta_{S}(\mathcal{G}-x)<\beta_{S}(\mathcal{G}) .
$$

Although the vertex set of a CG is itself a vertex covering set, the above findings reveal that in determining SVCSs, what is important is to find sets with the minimum number of vertices. In SVISs, finding a set with a maximum number of independent vertices is valuable. At the end of this section, the relationship between the SVCS and the SVIS is investigated.

Theorem 6. In CG $\mathcal{G}, F$ is an SVIS if and only if $V^{*} \backslash F$ is an SVCS.

Proof. Let $F$ be an SVIS. Then, there is no CSE between two vertices in F. Every CSE has at least one endpoint in $V^{*} \backslash F$. This means that $V^{*} \backslash F$ is an SVCS.

Conversely, if $F$ is an SVCS of $\mathcal{G}$, then, every CSE has at least one endpoint in $F$, so $V^{*} \backslash F$ is an SVIS.

Theorem 7. If $\mathcal{G}$ is a CG of order $p$ without an isolated vertex, then, $\alpha_{s}(\mathcal{G})+\beta_{S}(\mathcal{G})=p$.

Proof. Let $C$ and $F$ be two $\alpha_{\mathcal{S}}$-sets of $\mathcal{G}$, respectively. Thus, $V^{*} \backslash C$ is an SVCS and $V^{*} \backslash F$ is an SVIS. Therefore,

$$
p-\alpha_{\mathcal{S}}(\mathcal{G})=\left|V^{*} \backslash C\right| \leq \beta_{S}(\mathcal{G}), \quad p-\beta_{S}(\mathcal{G})=\left|V^{*} \backslash F\right| \geq \alpha_{S}(\mathcal{G}) .
$$

These two results imply that $\alpha_{\mathcal{S}}(\mathcal{G})+\beta_{\mathcal{S}}(\mathcal{G})=p$.

\section{Strong Vertex Covering Number in Operation on Two Cubic Graphs}

In this section, we studied some results of strong vertex covering number in certain operations on two cubic graphs. First, we determine the SVCS number in the union of two cubic graphs.

Definition 13. Consider $\mathcal{G}_{1}=\left(A_{1}, B_{1}\right)$ and $\mathcal{G}_{2}=\left(A_{2}, B_{2}\right)$ as the two CGs on $\mathcal{G}_{1}^{*}$ and $\mathcal{G}_{2}^{*}$, respectively. The union of two $C G s \mathcal{G}_{1}$ and $\mathcal{G}_{2}$ is shown by $\mathcal{G}_{1} \cup \mathcal{G}_{2}=\left(A_{1} \cup A_{2}, B_{1} \cup B_{2}\right)$ and is described as:

$$
(i)\left[\mu_{A_{1}}^{L} \cup \mu_{A_{2}}^{L}, \mu_{A_{1}}^{U} \cup \mu_{A_{2}}^{U}\right](x)= \begin{cases}{\left[\begin{array}{l}
\mu_{A_{1}}^{L}(x), \mu_{A_{1}}^{U}(x) \\
\mu_{A_{2}}^{L}(x), \mu_{A_{2}}^{U}(x)
\end{array}\right]} & \text { if } x \in V_{1}^{*}-V_{2}^{*} \\
\left.\mu_{A_{1}}^{L}(x) \vee \mu_{A_{2}}^{L}(x), \mu_{A_{1}}^{U}(x) \vee \mu_{A_{2}}^{U}(x)\right] & \text { if } x \in V_{2}^{*}-V_{1}^{*}\end{cases}
$$


(ii) $\left(\sigma_{A_{1}} \cup \sigma_{A_{2}}\right)(x)= \begin{cases}\sigma_{A_{1}}(x) & \text { if } x \in V_{1}^{*}-V_{2}^{*} \\ \sigma_{A_{2}}(x) & \text { if } x \in V_{2}^{*}-V_{1}^{*} \\ \sigma_{A_{1}}(x) \vee \sigma_{A_{2}}(x) & \text { if } x \in V_{1}^{*} \cap V_{2}^{*} .\end{cases}$

(iii) $\left[\mu_{B_{1}}^{L} \cup \mu_{B_{2}}^{L}, \mu_{B_{1}}^{U} \cup \mu_{B_{2}}^{U}\right](x y)= \begin{cases}{\left[\begin{array}{l}\mu_{B_{1}}^{L}(x y), \mu_{B_{1}}^{U}(x y) \\ \mu_{B_{2}}^{L}(x y), \mu_{B_{2}}^{U}(x y)\end{array}\right]} & \text { if } x y \in E_{1}^{*}-E_{2}^{*} \\ \left.\mu_{B_{1}}^{L}(x y) \vee \mu_{B_{2}}^{L}(x y), \mu_{B_{1}}^{U}(x y) \vee \mu_{B_{2}}^{U}(x y)\right] & \text { if } x y \in E_{2}^{*}-E_{1}^{*} \\ & \text { if } x y \in E_{1}^{*} \cap E_{2}^{*} .\end{cases}$

$(i v)\left(\sigma_{B_{1}} \cup \sigma_{B_{2}}\right)(x y)= \begin{cases}\sigma_{B_{1}}(x y) & \text { if } x y \in E_{1}^{*}-E_{2}^{*} \\ \sigma_{B_{2}}(x y) & \text { if } x y \in E_{2}^{*}-E_{1}^{*} \\ \sigma_{B_{1}}(x y) \vee \sigma_{B_{2}}(x y) & \text { if } x y \in E_{1}^{*} \cap E_{2}^{*} .\end{cases}$

Theorem 8. If $C_{1} \subseteq V_{1}^{*}$ and $C_{2} \subseteq V_{2}^{*}$ are two SVCSs of the CGs $\mathcal{G}_{1}=\left(A_{1}, B_{1}\right)$ and $\mathcal{G}_{2}=$ $\left(A_{2}, B_{2}\right)$, respectively, then, $C_{1} \cup C_{2}$ is the $S V C S$ of $\mathcal{G}_{1} \cup \mathcal{G}_{2}$.

Proof. Consider $C_{1}$ and $C_{2}$ as the two SVCSs of the CG $\mathcal{G}_{1}$ and $\mathcal{G}_{2}$, respectively. Therefore, every CSE is adjacent to at least one vertex in $C_{1}$ or $C_{2}$. The edges in the $\mathcal{G}_{1} \cup \mathcal{G}_{2}$ are $x y \in E_{1}^{*}$ or $x y \in E_{2}^{*}$. If $x y \in E_{1}$ is a CSE in the $\mathcal{G}_{1} \cup \mathcal{G}_{2}$, then, $C_{1}$ covers these edges since $C_{1}$ is an SVCS of $\mathcal{G}_{1}$. If $x y \in E_{2}^{*}$ is a CSE in $\mathcal{G}_{1} \cup \mathcal{G}_{2}$, then, $C_{2}$ covers these edges because $C_{2}$ is an SVCS of $\mathcal{G}_{2}$. Hence, $C_{1} \cup C_{2}$ is the SVCS of $\mathcal{G}_{1} \cup \mathcal{G}_{2}$.

Corollary 4. If the $C G \mathcal{G}_{1} \cup \mathcal{G}_{2}$ is the union of two $C G s \mathcal{G}_{1}$ and $\mathcal{G}_{2}$, then

$$
\alpha_{s}\left(\mathcal{G}_{1} \cup \mathcal{G}_{2}\right)=\left|C_{1} \cup C_{2}\right|,
$$

where $C_{1}$ and $C_{2}$ are SVCSs of CGs $\mathcal{G}_{1}$ and $\mathcal{G}_{2}$, respectively.

Example 4. Consider two strong CGs $\mathcal{G}_{1}$ and $\mathcal{G}_{2}$ as shown in Figure 3.

$C_{1}=\left\{y_{1}\right\}$ and $C_{2}=\left\{x_{2}\right\}$ are the SVCSs of $\mathcal{G}_{1}$ and $\mathcal{G}_{2}$, respectively. It is clear that $C_{1} \cup C_{2}=\left\{y_{1}, x_{2}\right\}$ is the SVCS of the CG $\mathcal{G}_{1} \cup \mathcal{G}_{2}$. Therefore,

$$
\alpha_{s}\left(\mathcal{G}_{1} \cup \mathcal{G}_{2}\right)=\left|C_{1} \cup C_{2}\right|=0.43+0.56=0.99 \text {. }
$$

$\langle[0.3,0.4], 0.6\rangle$

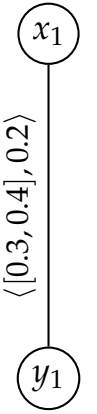

$\langle[0.6,0.7], 0.2\rangle$

$\mathcal{G}_{1}$

$$
\langle[0.4,0.6], 0.5\rangle
$$

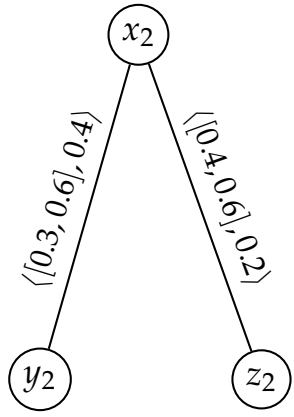

$\langle[0.3,0.7], 0.4\rangle \quad\langle[0.5,0.6], 0.2\rangle$

$\mathcal{G}_{2}$

$$
\langle[0.3,0.7], 0.4\rangle \quad\langle[0.5,0.6], 0.2\rangle
$$

$y_{2}$

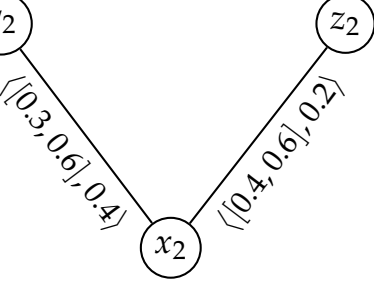

$\langle[0.4,0.6], 0.5\rangle$

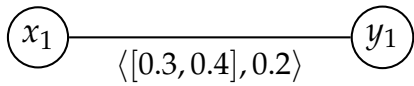

$\langle[0.3,0.4], 0.6\rangle \quad\langle[0.6,0.7], 0.2\rangle$

$\mathcal{G}_{1} \cup \mathcal{G}_{2}$

Figure 3. The union of $\mathcal{G}_{1}$ and $\mathcal{G}_{2}$.

Investigating and determining the strong vertex covering number in the joining of two cubic graphs are studied in the following. 
Definition 14. Consider $\mathcal{G}_{1}=\left(A_{1}, B_{1}\right)$ and $\mathcal{G}_{2}=\left(A_{2}, B_{2}\right)$ as the two CGs of $\mathcal{G}_{1}^{*}$ and $\mathcal{G}_{2}^{*}$, respectively. The joining of two $C G s \mathcal{G}_{1}$ and $\mathcal{G}_{2}$ is shown by $\mathcal{G}_{1}+\mathcal{G}_{2}=\left(A_{1}+A_{2}, B_{1}+B_{2}\right)$ and is described as:

$$
\begin{aligned}
& \text { (i) }\left\{\begin{array}{l}
{\left[\mu_{A_{1}}^{L}+\mu_{A_{2}}^{L}, \mu_{A_{1}}^{U}+\mu_{A_{2}}^{U}\right](x)=\left[\mu_{A_{1}}^{L} \cup \mu_{A_{2}}^{L} \mu_{A_{1}}^{U} \cup \mu_{A_{2}}^{U}\right](x)} \\
\left(\sigma_{A_{1}}+\sigma_{A_{2}}\right)(x)=\left(\sigma_{A_{1}} \cup \sigma_{A_{2}}\right)(x) \\
\text { for } x \in V_{1}^{*} \cup V_{2}^{*},
\end{array}\right. \\
& \text { (ii) }\left\{\begin{array}{l}
{\left[\mu_{B_{1}}^{L}+\mu_{B_{2}}^{L}, \mu_{B_{1}}^{U}+\mu_{B_{2}}^{U}\right](x y)=\left[\mu_{B_{1}}^{L} \cup \mu_{B_{2}}^{L}, \mu_{B_{1}}^{U} \cup \mu_{B_{2}}^{U}\right](x y)} \\
\left(\sigma_{B_{1}}+\sigma_{B_{2}}\right)(x y)=\left(\sigma_{B_{1}} \cup \sigma_{B_{2}}\right)(x y) \\
\text { for } x y \in E_{1}^{*} \cap E_{2}^{*},
\end{array}\right. \\
& \text { (iii) }\left\{\begin{array}{l}
{\left[\mu_{B_{1}}^{L}+\mu_{B_{2}}^{L}, \mu_{B_{1}}^{U}+\mu_{B_{2}}^{U}\right](x y)=\left[\mu_{A_{1}}^{L}(x) \wedge \mu_{A_{1}}^{L}(y), \mu_{A_{2}}^{U}(x) \wedge \mu_{A_{2}}^{U}(y)\right]} \\
\left(\sigma_{B_{1}}+\sigma_{B_{2}}\right)(x y)=\sigma_{A_{1}}(x) \wedge \sigma_{A_{2}}(y)
\end{array}\right.
\end{aligned}
$$

$x y$ belongs to the set of all edges joining the vertices of $V_{1}^{*}$ and $V_{2}^{*}$.

Theorem 9. If $C_{1}$ and $C_{2}$ are two SVCSs of the CGs $\mathcal{G}_{1}=\left(A_{1}, B_{1}\right)$ and $\mathcal{G}_{2}=\left(A_{2}, B_{2}\right)$, respectively, then, $V_{1}^{*} \cup C_{2}$ and $C_{1} \cup V_{2}^{*}$ are the $S V C S$ of $\mathcal{G}_{1}+\mathcal{G}_{2}$.

Proof. Consider $C_{1}$ and $C_{2}$ as the two SVCSs of the CGs $\mathcal{G}_{1}$ and $\mathcal{G}_{2}$, respectively. Therefore, every CSE is adjacent to at least one vertex in $C_{1}$ or $C_{2}$.

There are the following cases for the edge $x y$ in the $C G \mathcal{G}_{1}+\mathcal{G}_{2}$.

Case (i). If $x y \in E_{1}^{*}$ is a CSE in $\mathcal{G}_{1}+\mathcal{G}_{2}$, then, $C_{1}$ covers this edge since $C_{1}$ is an SVCS of $G_{1}$. Case (ii). If $x y \in E_{2}^{*}$ is a CSE in $\mathcal{G}_{1}+\mathcal{G}_{2}$, then, $C_{2}$ covers this edge since $C_{2}$ is an SVCS of $\mathcal{G}_{2}$. Case (iii). If $x y \in \mathcal{G}_{1}+\mathcal{G}_{2}, x \in V_{1}^{*}$ and $y \in V_{2}^{*}$, then this edge is CSE in $\mathcal{G}_{1}+\mathcal{G}_{2}$ since this edge is adjacent to $V_{1}^{*}$ and $V_{2}^{*}$. This implies that $V_{1}^{*}$ and $V_{2}^{*}$ covered these edges in $\mathcal{G}_{1}+\mathcal{G}_{2}$. The above cases imply that the CSEs in $\mathcal{G}_{1}+\mathcal{G}_{2}$ are covered by the set $V_{1}^{*} \cup C_{2}$ and $C_{1} \cup V_{2}^{*}$.

Corollary 5. If the $C G \mathcal{G}_{1}+\mathcal{G}_{2}$ is the joining of two $C G s \mathcal{G}_{1}$ and $\mathcal{G}_{2}$, then,

$$
\alpha_{s}\left(\mathcal{G}_{1}+\mathcal{G}_{2}\right)=\left|V_{1}^{*} \cup C_{2}\right| \wedge\left|C_{1} \cup V_{2}^{*}\right|,
$$

where $C_{1}$ and $C_{2}$ are two $\alpha_{s}$-sets of $C G s \mathcal{G}_{1}$ and $\mathcal{G}_{2}$, respectively.

Example 5. Consider two CGs $\mathcal{G}_{1}$ and $\mathcal{G}_{2}$ of the Example 4. The joining of $\mathcal{G}_{1}$ and $\mathcal{G}_{2}$ is drawn in Figure 4.

By routine calculations, we have

$$
C_{1}=\left\{y_{1}\right\}, \quad C_{2}=\left\{x_{2}\right\}, \quad V_{1}^{*} \cup C_{2}=\left\{x_{1}, x_{2}, y_{1}\right\}, \quad C_{1} \cup V_{2}^{*}=\left\{y_{1}, x_{2}, y_{2}, z_{2}\right\} .
$$

Therefore,

$$
\begin{aligned}
\alpha_{S}\left(\mathcal{G}_{1}+\mathcal{G}_{2}\right) & =\min \{(0.56+0.56+0.43),(0.43+0.56+0.6+0.43)\} \\
& =\min \{1.55,2.45\}=1.55
\end{aligned}
$$




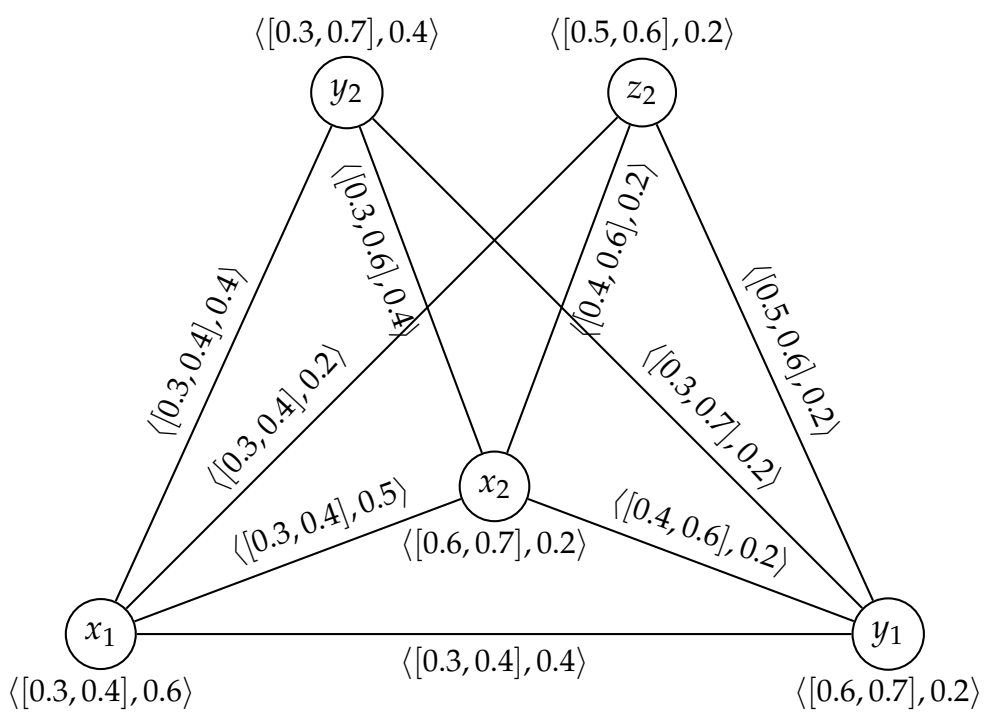

Figure 4. The joining of $\mathcal{G}_{1}$ and $\mathcal{G}_{2}$.

In the following, the strong vertex covering number in the direct product operation is investigated.

Definition 15. Consider $\mathcal{G}_{1}=\left(A_{1}, B_{1}\right)$ and $\mathcal{G}_{2}=\left(A_{2}, B_{2}\right)$ as the two CGs with underlying $\mathcal{G}_{1}^{*}$ and $\mathcal{G}_{2}^{*}$, respectively, where $V_{1}^{*} \cap V_{2}^{*}=\varnothing$. The direct product of $\mathcal{G}_{1}$ and $\mathcal{G}_{2}$ is shown by $\mathcal{G}_{1} \cdot \mathcal{G}_{2}=\left(A_{1} \cdot A_{2}, B_{1} \cdot B_{2}\right)$ and is described as:

(i) $\left\{\begin{array}{l}{\left[\mu_{A_{1}}^{L} \cdot \mu_{A_{2}}^{L} \mu_{A_{1}}^{U} \cdot \mu_{A_{2}}^{U}\right]\left(x_{1}, x_{2}\right)=\left[\mu_{A_{1}}^{L}\left(x_{1}\right) \wedge \mu_{A_{2}}^{L}\left(x_{2}\right), \mu_{A_{1}}^{U}\left(x_{1}\right) \wedge \mu_{A_{2}}^{U}\left(x_{2}\right)\right]} \\ \left(\sigma_{A_{1}} \cdot \sigma_{A_{2}}\right)\left(x_{1}, x_{2}\right)=\sigma_{A_{1}}\left(x_{1}\right) \wedge \sigma_{A_{2}}\left(x_{2}\right) \\ \forall\left(x_{1}, x_{2}\right) \in V_{1}^{*} \times V_{2}^{*},\end{array}\right.$

(ii) $\begin{cases} & {\left[\mu_{B_{1}}^{L} \cdot \mu_{B_{2}}^{L}, \mu_{B_{1}}^{U} \cdot \mu_{B_{2}}^{U}\right]\left(\left(x_{1}, x_{2}\right)\left(y_{1}, y_{2}\right)\right)=\left[\mu_{B_{1}}^{L}\left(x_{1} y_{1}\right) \wedge \mu_{B_{2}}^{L}\left(x_{2} y_{2}\right), \mu_{B_{1}}^{U}\left(x_{1} y_{1}\right) \wedge \mu_{B_{2}}^{U}\left(x_{2} y_{2}\right)\right]} \\ & \left(\sigma_{B_{1}} \cdot \sigma_{B_{2}}\right)\left(\left(x_{1}, x_{2}\right)\left(y_{1}, y_{2}\right)\right)=\sigma_{B_{1}}\left(x_{1} y_{1}\right) \wedge \sigma_{B_{2}}\left(x_{2} y_{2}\right) \\ \forall & \forall x_{1} y_{1} \in E_{1}^{*}, x_{2} y_{2} \in E_{2}^{*} .\end{cases}$

Theorem 10. If $C_{1}$ and $C_{2}$ are two SVCSs of the CGs $\mathcal{G}_{1}=\left(A_{1}, B_{1}\right)$ and $\mathcal{G}_{2}=\left(A_{2}, B_{2}\right)$, respectively, then, $V_{1}^{*} \times C_{2}$ or $C_{1} \times V_{2}^{*}$ is the minimum $S V C S$ of $\mathcal{G}_{1} \cdot \mathcal{G}_{2}$.

Proof. Consider $\mathcal{G}_{1}=\left(A_{1}, B_{1}\right)$ and $\mathcal{G}_{2}=\left(A_{2}, B_{2}\right)$ as the two CGs with two SVCSs $C_{1}$ and $C_{2}$, respectively. Suppose $x_{1} y_{1} \in E_{1}^{*}$ and $x_{2} y_{2} \in E_{2}^{*}$ to be the CSEs in $\mathcal{G}_{1}$ and $\mathcal{G}_{2}$, respectively. Therefore

$$
\begin{aligned}
\left(\mu_{B_{1}}^{L} \cdot \mu_{B_{2}}^{L}\right)\left(\left(x_{1}, x_{2}\right)\left(y_{1}, y_{2}\right)\right) & =\mu_{B_{1}}^{L}\left(x_{1} y_{1}\right) \wedge \mu_{B_{2}}^{L}\left(x_{2} y_{2}\right) \\
& =\mu_{A_{1}}^{L}\left(x_{1}\right) \wedge \mu_{A_{1}}^{L}\left(y_{1}\right) \wedge \mu_{A_{2}}^{L}\left(x_{2}\right) \wedge \mu_{A_{2}}^{L}\left(y_{2}\right) \\
& =\left(\mu_{A_{1}}^{L} \cdot \mu_{A_{2}}^{L}\right)\left(x_{1}, x_{2}\right) \wedge\left(\mu_{A_{1}}^{L} \cdot \mu_{A_{2}}^{L}\right)\left(y_{1}, y_{2}\right) .
\end{aligned}
$$

Similarly, $\left(\mu_{B_{1}}^{U} \cdot \mu_{B_{2}}^{U}\right)\left(\left(x_{1}, x_{2}\right)\left(y_{1}, y_{2}\right)\right)=\left(\mu_{A_{1}}^{U} \cdot \mu_{A_{2}}^{U}\right)\left(x_{1}, x_{2}\right) \wedge\left(\mu_{A_{1}}^{U} \cdot \mu_{A_{2}}^{U}\right)\left(y_{1}, y_{2}\right)$. Additionally,

$$
\begin{aligned}
\left(\sigma_{B_{1}} \cdot \sigma_{B_{2}}\right)\left(\left(x_{1}, x_{2}\right)\left(y_{1}, y_{2}\right)\right) & =\sigma_{B_{1}}\left(x_{1} y_{1}\right) \wedge \sigma_{B_{2}}\left(x_{2} y_{2}\right) \\
& =\sigma_{A_{1}}\left(x_{1}\right) \wedge \sigma_{A_{1}}\left(y_{1}\right) \wedge \sigma_{A_{2}}\left(x_{2}\right) \wedge \sigma_{A_{2}}\left(y_{2}\right) \\
& =\left(\sigma_{A_{1}} \cdot \sigma_{A_{2}}\right)\left(x_{1}, x_{2}\right) \wedge\left(\sigma_{A_{1}} \cdot \sigma_{A_{2}}\right)\left(y_{1}, y_{2}\right) .
\end{aligned}
$$

This implies that the edge $\left(\left(x_{1}, x_{2}\right)\left(y_{1}, y_{2}\right)\right)$ is a CSE in $\mathcal{G}_{1} \cdot \mathcal{G}_{2}$, which indicates that the end vertices of the $\left(\left(x_{1}, x_{2}\right)\left(y_{1}, y_{2}\right)\right)$ edge are at the $V_{1}^{*} \times C_{2}$ or $C_{1} \times V_{2}^{*}$. Therefore, $V_{1}^{*} \times C_{2}$ or $C_{1} \times V_{2}^{*}$ is the minimum SVCS of $\mathcal{G}_{1} \cdot \mathcal{G}_{2}$. 
Corollary 6. If the $C G \mathcal{G}_{1} \cdot \mathcal{G}_{2}$ is the direct product of two $C G s \mathcal{G}_{1}$ and $\mathcal{G}_{2}$, then,

$$
\alpha_{s}\left(\mathcal{G}_{1} \cdot \mathcal{G}_{2}\right)=\left|V_{1}^{*} \times C_{2}\right| \wedge\left|C_{1} \times V_{2}^{*}\right|
$$

Example 6. Consider the two CGs $\mathcal{G}_{1}$ and $\mathcal{G}_{2}$ and their direct product $\mathcal{G}_{1} \cdot \mathcal{G}_{2}$ as drawn in Figure 5. We have

$$
\begin{aligned}
& \left|V_{1}^{*} \times C_{2}\right|=\left|\left\{x_{1}, y_{1}\right\} \times\left\{y_{2}\right\}\right|=\left|\left\{x_{1} y_{2}, y_{1} y_{2}\right\}\right|=0.56+0.53=1.09 \\
& \left|C_{2} \times V_{2}^{*}\right|=\left|\left\{x_{1}\right\} \times\left\{x_{2}, y_{2}\right\}\right|=\left|\left\{x_{1} x_{2}, x_{1} y_{2}\right\}\right|=0.56+0.56=1.12
\end{aligned}
$$

Therefore, $\alpha_{s}\left(\mathcal{G}_{1} \cdot \mathcal{G}_{2}\right)=\min \{1.09,1.12\}=1.09$.

$\langle[0.2,0.6], 0.3\rangle$

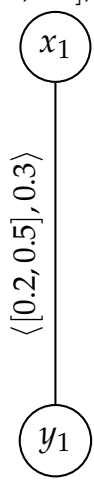

$\langle[0.3,0.5], 0.6\rangle$

$\mathcal{G}_{1}$ $\langle[0.4,0.7], 0.5\rangle$

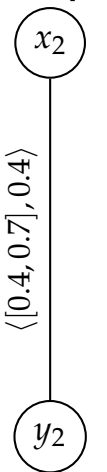

$\langle[0.7,0.9], 0.4\rangle$

$\mathcal{G}_{2}$ $\langle[0.2,0.6], 0.3\rangle$

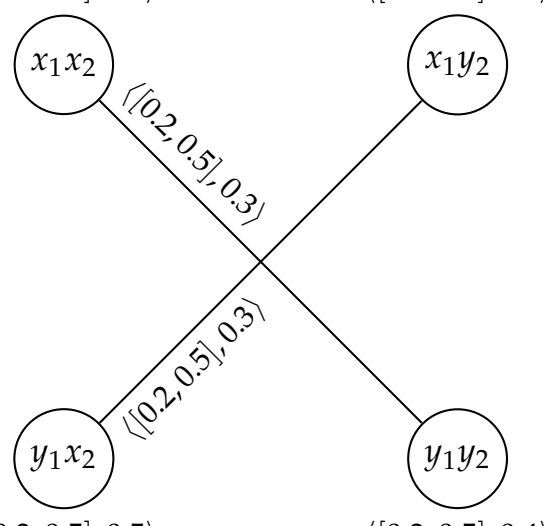

$\langle[0.3,0.5], 0.5\rangle$ $\langle[0.2,0.6], 0.3\rangle$

$\langle[0.3,0.5], 0.4\rangle$

$\mathcal{G}_{1} \cdot \mathcal{G}_{2}$

Figure 5. The direct product $\mathcal{G}_{1}$ and $\mathcal{G}_{2}$.

Next, the strong vertex covering number in a semi-strong product is determined.

Definition 16. If $\mathcal{G}_{1}=\left(A_{1}, B_{1}\right)$ and $\mathcal{G}_{2}=\left(A_{2}, B_{2}\right)$ are the two CGs on $\mathcal{G}_{1}^{*}$ and $\mathcal{G}_{2}^{*}$, respectively, then, the semi-strong product of $\mathcal{G}_{1}$ and $\mathcal{G}_{2}$ is denoted by $\mathcal{G}_{1} \bullet \mathcal{G}_{2}=\left(A_{1} \bullet A_{2}, B_{1} \bullet B_{2}\right)$ and is described as:

(i) $\left\{\begin{array}{l}{\left[\mu_{A_{1}}^{L} \bullet \mu_{A_{2}}^{L}, \mu_{A_{1}}^{U} \bullet \mu_{A_{2}}^{U}\right]\left(x_{1}, x_{2}\right)=\left[\mu_{A_{1}}^{L}\left(x_{1}\right) \wedge \mu_{A_{2}}^{L}\left(x_{2}\right), \mu_{A_{1}}^{U}\left(x_{1}\right) \wedge \mu_{A_{2}}^{U}\left(x_{2}\right)\right]} \\ \left(\sigma_{A_{1}} \bullet \sigma_{A_{2}}\right)\left(x_{1}, x_{2}\right)=\sigma_{A_{1}}\left(x_{1}\right) \wedge \sigma_{A_{2}}\left(x_{2}\right) \\ \forall\left(x_{1}, x_{2}\right) \in V_{1}^{*} \times V_{2}^{*},\end{array}\right.$

(ii) $\left\{\begin{array}{l}{\left[\mu_{B_{1}}^{L} \bullet \mu_{B_{2}}^{L}, \mu_{B_{1}}^{U} \bullet \mu_{B_{2}}^{U}\right]\left(\left(z, x_{2}\right)\left(z, y_{2}\right)\right)=\left[\mu_{A_{1}}^{L}(z) \wedge \mu_{B_{2}}^{L}\left(x_{2} y_{2}\right), \mu_{A_{1}}^{U}(z) \wedge \mu_{B_{2}}^{U}\left(x_{2} y_{2}\right)\right]} \\ \left(\sigma_{B_{1}} \bullet \sigma_{B_{2}}\right)\left(\left(z, x_{2}\right)\left(z, y_{2}\right)\right)=\sigma_{A_{1}}(z) \wedge \sigma_{B_{2}}\left(x_{2} y_{2}\right) \\ \forall z \in V_{1}^{*}, x_{2} y_{2} \in E_{2}^{*}\end{array}\right.$

(iii) $\left\{\begin{array}{l}{\left[\mu_{B_{1}}^{L} \bullet \mu_{B_{2}}^{L}, \mu_{B_{1}}^{U} \bullet \mu_{B_{2}}^{U}\right]\left(\left(x_{1}, x_{2}\right)\left(y_{1}, y_{2}\right)\right)=\left[\mu_{B_{1}}^{L}\left(x_{1} y_{1}\right) \wedge \mu_{B_{2}}^{L}\left(x_{2} y_{2}\right), \mu_{B_{1}}^{U}\left(x_{1} y_{1}\right) \wedge \mu_{B_{2}}^{U}\left(x_{2} y_{2}\right)\right]} \\ \left(\sigma_{B_{1}} \bullet \sigma_{B_{2}}\right)\left(\left(x_{1}, x_{2}\right)\left(y_{1}, y_{2}\right)\right)=\sigma_{B_{1}}\left(x_{1} y_{1}\right) \wedge \sigma_{B_{2}}\left(x_{2} y_{2}\right) \\ \forall x_{1} y_{1} \in E_{1}^{*}, x_{2} y_{2} \in E_{2}^{*}\end{array}\right.$

Theorem 11. Let $C_{1}$ and $C_{2}$ be the SVCSs of the CGs $\mathcal{G}_{1}=\left(A_{1}, B_{1}\right)$ and $\mathcal{G}_{2}=\left(A_{2}, B_{2}\right)$, respectively. Then, $V_{1}^{*} \times C_{2}$ is the $S V C S$ of $\mathcal{G}_{1} \cdot \mathcal{G}_{2}$.

Proof. Let $C_{1}$ and $C_{2}$ be the SVCSs of the CGs $\mathcal{G}_{1}$ and $\mathcal{G}_{2}$, respectively. Therefore, every CSE is adjacent to at least one vertex in $C_{1}$ or $C_{2}$. The following cases exist for the edges of $\mathcal{G}_{1} \cdot \mathcal{G}_{2}$. 
Case (i). $\left(z, x_{2}\right)\left(z, y_{2}\right)$ if $z \in V_{1}^{*}, x_{2} y_{2} \in E_{2}^{*}$.

$$
\begin{aligned}
\left(\mu_{B_{1}}^{L} \cdot \mu_{B_{2}}^{L}\right)\left(\left(z, x_{2}\right)\left(z, y_{2}\right)\right) & =\mu_{A_{1}}^{L}(z) \wedge \mu_{B_{1}}^{L}\left(x_{2} y_{2}\right) \\
& =\mu_{A_{1}}^{L}(z) \wedge \mu_{A_{2}}^{L}\left(x_{2}\right) \wedge \mu_{A_{2}}^{L}\left(y_{2}\right) \\
& =\mu_{A_{1}}^{L}(z) \wedge \mu_{A_{2}}^{L}\left(x_{2}\right) \wedge \mu_{A_{1}}^{L}\left(y_{1}\right) \wedge \mu_{A_{2}}^{L}\left(y_{2}\right) \\
& =\left(\mu_{A_{1}}^{L} \cdot \mu_{A_{2}}^{L}\right)\left(z, x_{2}\right) \wedge\left(\mu_{A_{1}}^{L} \cdot \mu_{A_{2}}^{L}\right)\left(y_{1}, y_{2}\right) .
\end{aligned}
$$
tionally,

Similarly, $\left(\mu_{B_{1}}^{U} \cdot \mu_{B_{2}}^{U}\right)\left(\left(z, x_{2}\right)\left(z, y_{2}\right)\right)=\left(\mu_{A_{1}}^{U} \cdot \mu_{A_{2}}^{U}\right)\left(z, x_{2}\right) \wedge\left(\mu_{A_{1}}^{U} \cdot \mu_{A_{2}}^{U}\right)\left(y_{1}, y_{2}\right)$. Addi-

$$
\begin{aligned}
\left(\sigma_{B_{1}} \cdot \sigma_{B_{2}}\right)\left(\left(z, x_{2}\right)\left(z y_{2}\right)\right) & =\sigma_{A_{1}}(z) \wedge \sigma_{B_{2}}\left(x_{2} y_{2}\right) \\
& =\sigma_{A_{1}}(z) \wedge \sigma_{A_{2}}\left(x_{2}\right) \wedge \sigma_{A_{2}}\left(y_{2}\right) \\
& =\sigma_{A_{1}}(z) \wedge \sigma_{A_{2}}\left(x_{2}\right) \wedge \sigma_{A_{1}}\left(y_{1}\right) \wedge \sigma_{A_{2}}\left(y_{2}\right) \\
& =\left(\sigma_{A_{1}} \cdot \sigma_{A_{2}}\right)\left(z, x_{2}\right) \wedge\left(\sigma_{A_{1}} \cdot \sigma_{A_{2}}\right)\left(y_{1}, y_{2}\right) .
\end{aligned}
$$

Therefore, the edge $\left(z, x_{2}\right)\left(z, y_{2}\right)$ is a CSE in the CG $\mathcal{G}_{1} \cdot \mathcal{G}_{2}$. Therefore, $\left(z, x_{2}\right) \in V_{1}^{*} \times C_{2}$ or $\left(z, y_{2}\right) \in V_{1}^{*} \times C_{2}$

Case (ii). $\left(x_{1}, x_{2}\right)\left(y_{1}, y_{2}\right)$ if $x_{1} y_{1} \in E_{1}^{*}$ and $x_{2} y_{2} \in E_{2}^{*}$. If $x_{1} y_{1} \in E_{1}^{*}$ and $x_{2} y_{2} \in E_{2}^{*}$ are a CSEs, then, we have

$$
\begin{aligned}
\left(\mu_{B_{1}}^{L} \cdot \mu_{B_{2}}^{L}\right)\left(\left(x_{1}, x_{2}\right)\left(y_{1}, y_{2}\right)\right) & =\mu_{B_{1}}^{L}\left(x_{1} y_{1}\right) \wedge \mu_{B_{2}}^{L}\left(x_{2} y_{2}\right) \\
& =\mu_{A_{1}}^{L}\left(x_{1}\right) \wedge \mu_{A_{2}}^{L}\left(x_{2}\right) \wedge \mu_{A_{1}}^{L}\left(y_{1}\right) \wedge \mu_{A_{2}}^{L}\left(y_{2}\right) \\
& =\left(\mu_{A_{1}}^{L} \cdot \mu_{A_{2}}^{L}\right)\left(x_{1}, x_{2}\right) \wedge\left(\mu_{A_{1}}^{L} \cdot \mu_{A_{2}}^{L}\right)\left(y_{1}, y_{2}\right) .
\end{aligned}
$$
ditionally,

Similarly, $\left(\mu_{B_{1}}^{U} \cdot \mu_{B_{2}}^{U}\right)\left(\left(x_{1}, x_{2}\right)\left(y_{1}, y_{2}\right)\right)=\left(\mu_{A_{1}}^{U} \cdot \mu_{A_{2}}^{U}\right)\left(x_{1}, x_{2}\right) \wedge\left(\mu_{A_{1}}^{U} \cdot \mu_{A_{2}}^{U}\right)\left(y_{1}, y_{2}\right)$. Ad-

$$
\begin{aligned}
\left(\sigma_{B_{1}} \cdot \sigma_{B_{2}}\right)\left(\left(x_{1}, x_{2}\right)\left(y_{1} y_{2}\right)\right) & =\sigma_{B_{1}}\left(x_{1} y_{1}\right) \wedge \sigma_{B_{2}}\left(x_{2} y_{2}\right) \\
& =\sigma_{A_{1}}\left(x_{1}\right) \wedge \sigma_{A_{2}}\left(x_{2}\right) \wedge \sigma_{A_{1}}\left(y_{1}\right) \wedge \sigma_{A_{2}}\left(y_{2}\right) \\
& =\left(\sigma_{A_{1}} \cdot \sigma_{A_{2}}\right)\left(x_{1}, x_{2}\right) \wedge\left(\sigma_{A_{1}} \cdot \sigma_{A_{2}}\right)\left(y_{1}, y_{2}\right) .
\end{aligned}
$$

This implies that the edge $\left(x_{1}, x_{2}\right)\left(y_{1}, y_{2}\right)$ is a CSE in $\mathcal{G}_{1} \cdot \mathcal{G}_{2}$. Since the edge $x_{2} y_{2}$ is a CSE in $\mathcal{G}_{2}$, then $x_{2} \in C_{2}$ or $y_{2} \in C_{2}$, therefore, $\left(x_{1}, y_{2}\right) \in V_{1}^{*} \times C_{2}$ or $\left(y_{1}, y_{2}\right) \in V_{1}^{*} \times C_{2}$. Thus, the set $V_{1}^{*} \times C_{2}$ is covering all the CSEs in $\mathcal{G}_{1} \cdot \mathcal{G}_{2}$.

Corollary 7. If the CG $\mathcal{G}_{1} \cdot \mathcal{G}_{2}$ is the semi-strong product of two CGs $\mathcal{G}_{1}$ and $\mathcal{G}_{2}$, then, $\alpha_{s}\left(\mathcal{G}_{1}\right.$. $\left.\mathcal{G}_{2}\right)=\left|V_{1}^{*} \times C_{2}\right|$.

Example 7. Consider two CGs $\mathcal{G}_{1}$ and $\mathcal{G}_{2}$ as drawn in Example 6. The semi-strong product of $\mathcal{G}_{1}$ and $\mathcal{G}_{2}$ is shown in Figure 6.

The minimum SVCS is $V_{1}^{*} \times C_{2}$. Therefore,

$$
\alpha_{s}\left(\mathcal{G}_{1} \cdot G_{2}\right)=\left|V_{1}^{*} \times C_{2}\right|=\mid\left\{x_{1} y_{2}, y_{1} y_{2}\right\}=0.56+0.53=1.09 .
$$




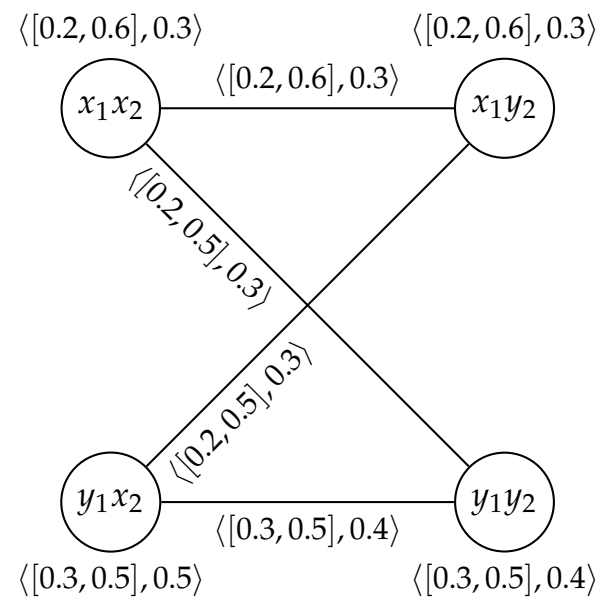

Figure 6. The semi-strong product $\mathcal{G}_{1}$ and $\mathcal{G}_{2}$.

The following definition gives the strong vertex covering number in the Cartesian product.

Definition 17. Consider $\mathcal{G}_{1}=\left(A_{1}, B_{1}\right)$ and $\mathcal{G}_{2}=\left(A_{2}, B_{2}\right)$ as the two CGs of the underlying graphs $\mathcal{G}_{1}^{*}$ and $\mathcal{G}_{2}^{*}$, respectively. The Cartesian product of $\mathcal{G}_{1}$ and $\mathcal{G}_{2}$ is shown by $\mathcal{G}_{1} \times \mathcal{G}_{2}=$ $\left(A_{1} \times A_{2}, B_{1} \times B_{2}\right)$ and is described as:

(i) $\left\{\begin{array}{l}{\left[\mu_{A_{1}}^{L} \times \mu_{A_{2}}^{L}, \mu_{A_{1}}^{U} \times \mu_{A_{2}}^{U}\right]\left(x_{1}, x_{2}\right)=\left[\mu_{A_{1}}^{L}\left(x_{1}\right) \wedge \mu_{A_{2}}^{L}\left(x_{2}\right), \mu_{A_{1}}^{U}\left(x_{1}\right) \wedge \mu_{A_{2}}^{U}\left(x_{2}\right)\right]} \\ \left(\sigma_{A_{1}} \times \sigma_{A_{2}}\right)\left(x_{1}, x_{2}\right)=\sigma_{A_{1}}\left(x_{1}\right) \wedge \sigma_{A_{2}}\left(x_{2}\right), \quad \forall\left(x_{1}, x_{2}\right) \in V_{1}^{*} \times V_{2}^{*},\end{array}\right.$

(ii) $\left\{\begin{array}{l}{\left[\mu_{B_{1}}^{L} \times \mu_{B_{2}}^{L}, \mu_{B_{1}}^{U} \times \mu_{B_{2}}^{U}\right]\left(\left(x, x_{2}\right)\left(x, y_{2}\right)\right)=\left[\mu_{A_{1}}^{L}(x) \wedge \mu_{B_{2}}^{L}\left(x_{2} y_{2}\right), \mu_{A_{1}}^{U}(x) \wedge \mu_{B_{2}}^{U}\left(x_{2} y_{2}\right)\right]} \\ \left(\sigma_{B_{1}} \times \sigma_{B_{2}}\right)\left(\left(x, x_{2}\right)\left(x, y_{2}\right)=\sigma_{A_{1}}(x) \wedge \sigma_{B_{2}}\left(x_{2} y_{2}\right), \forall x \in V_{1}^{*}, x_{2} y_{2} \in E_{2}^{*},\right.\end{array}\right.$

(iii) $\left\{\begin{array}{l}{\left[\mu_{B_{1}}^{L} \times \mu_{B_{2}}^{L}, \mu_{B_{1}}^{U} \times \mu_{B_{2}}^{U}\right]\left(\left(x_{1}, x\right)\left(y_{1}, x\right)\right)=\left[\mu_{B_{1}}^{L}\left(x_{1} y_{1}\right) \wedge \mu_{A_{2}}^{L}(x), \mu_{B_{1}}^{U}\left(x_{1} y_{1}\right) \wedge \mu_{A_{2}}^{U}(x)\right]} \\ \left(\sigma_{B_{1}} \times \sigma_{B_{2}}\right)\left(\left(x_{1}, x\right)\left(y_{1}, x\right)=\sigma_{B_{1}}\left(x_{1} y_{1}\right) \wedge \sigma_{A_{2}}(x), \forall x \in V_{2}^{*}, x_{1} y_{1} \in E_{1}^{*} .\right.\end{array}\right.$

Theorem 12. Let $C_{1}$ and $C_{2}$ be the SVCSs of the CGs $\mathcal{G}_{1}=\left(A_{1}, B_{1}\right)$ and $\mathcal{G}_{2}=\left(A_{2}, B_{2}\right)$, respectively. Then, $\left\{C_{1} \times\left(V_{2}^{*} \backslash C_{2}\right)\right\} \cup\left\{\left(V_{1}^{*} \backslash C_{1}\right) \times C_{2}\right\}$ is the SVCS of $\mathcal{G}_{1} \times \mathcal{G}_{2}$.

Proof. Let $\mathcal{G}_{1}$ and $\mathcal{G}_{2}$ be two CGs with the SVCSs $C_{1}$ and $C_{2}$, respectively.

Case (i). $x \in V_{1}^{*}$ and $x_{2} y_{2} \in E_{2}^{*}$.

If the edge $x_{2} y_{2} \in E_{2}^{*}$ is a CSE in $\mathcal{G}_{2}$, then,

$$
\begin{aligned}
\left(\mu_{B_{1}}^{L} \times \mu_{B_{2}}^{L}\right)\left(\left(x, x_{2}\right)\left(x, y_{2}\right)\right) & =\mu_{A_{1}}^{L}(x) \wedge \mu_{B_{2}}^{L}\left(x_{2} y_{2}\right) \\
& =\mu_{A_{1}}^{L}(x) \wedge \mu_{A_{2}}^{L}\left(x_{2}\right) \wedge \mu_{A_{2}}^{L}\left(y_{2}\right) \\
& =\mu_{A_{1}}^{L}(x) \wedge \mu_{A_{2}}^{L}\left(x_{2}\right) \wedge \mu_{A_{1}}^{L}(x) \wedge \mu_{A_{2}}^{L}\left(y_{2}\right) \\
& =\left(\mu_{A_{1}}^{L} \times \mu_{A_{2}}^{L}\right)\left(x, x_{2}\right) \wedge\left(\mu_{A_{1}}^{L} \times \mu_{A_{2}}^{L}\right)\left(x, y_{2}\right) .
\end{aligned}
$$
ditionally,

Similarly, $\left(\mu_{B_{1}}^{U} \times \mu_{B_{2}}^{U}\right)\left(\left(x, x_{2}\right)\left(x, y_{2}\right)\right)=\left(\mu_{A_{1}}^{U} \times \mu_{A_{2}}^{U}\right)\left(x, x_{2}\right) \wedge\left(\mu_{A_{1}}^{U} \times \mu_{A_{2}}^{U}\right)\left(x, y_{2}\right)$. Ad-

$$
\begin{aligned}
\left(\sigma_{B_{1}} \times \sigma_{B_{2}}\right)\left(\left(x, x_{2}\right)\left(x, y_{2}\right)\right) & =\sigma_{A_{1}}(x) \wedge \sigma_{B_{2}}\left(x_{2} y_{2}\right) \\
& =\sigma_{A_{1}}(x) \wedge \sigma_{A_{2}}\left(x_{2}\right) \wedge \sigma_{A_{2}}\left(y_{2}\right) \\
& =\sigma_{A_{1}}(x) \wedge \sigma_{A_{2}}\left(x_{2}\right) \wedge \sigma_{A_{2}}(x) \wedge \sigma_{A_{2}}\left(y_{2}\right) \\
& =\left(\sigma_{A_{1}} \times \sigma_{A_{2}}\right)\left(x, x_{2}\right) \wedge\left(\sigma_{A_{1}} \times \sigma_{A_{2}}\right)\left(x, y_{2}\right) .
\end{aligned}
$$


This implies that the edge $\left(x, x_{2}\right)\left(x, y_{2}\right)$ is a CSE in $\mathcal{G}_{1} \times \mathcal{G}_{2}$. Since $x_{2} y_{2}$ is a CSE in $\mathcal{G}_{2}$, then, $x_{2} \in C_{2}$ or $y_{2} \in C_{2}$ therefore, the vertices $\left(x, x_{2}\right) \in\left(V_{1}^{*} \backslash C_{1}\right) \times C_{2}$ or $\left(x y_{2}\right) \in$ $\left(V_{1}^{*} \backslash C_{1}\right) \times C_{2}$ in $\mathcal{G}_{1} \times \mathcal{G}_{2}$. Thus, the CSEs of this form are covered by the set $\left(V_{1}^{*} \backslash C_{1}\right) \times C_{2}$. Case (ii). $x \in V_{2}^{*}$ and $x_{1} y_{1} \in E_{1}^{*}$.

If $x_{1} y_{1} \in E_{1}^{*}$ is a $\operatorname{CSE}$ in $\mathcal{G}_{1}$, then,

$$
\begin{aligned}
\left(\mu_{B_{1}}^{L} \times \mu_{B_{2}}^{L}\right)\left(\left(x_{1}, x\right)\left(y_{1}, x\right)\right) & =\mu_{B_{1}}^{L}\left(x_{1} y_{1}\right) \wedge \mu_{A_{2}}^{L}(x) \\
& =\mu_{A_{1}}^{L}\left(x_{1}\right) \wedge \mu_{A_{1}}^{L}\left(y_{1}\right) \wedge \mu_{A_{2}}^{L}(x) \\
& =\mu_{A_{1}}^{L}\left(x_{1}\right) \wedge \mu_{A_{2}}^{L}(x) \wedge \mu_{A_{1}}^{L}\left(y_{1}\right) \wedge \mu_{A_{2}}^{L}(x) \\
& =\left(\mu_{A_{1}}^{L} \times \mu_{A_{2}}^{L}\right)\left(x_{1}, x\right) \wedge\left(\mu_{A_{1}}^{L} \times \mu_{A_{2}}^{L}\right)\left(y_{1}, x\right) .
\end{aligned}
$$
ditionally,

Similarly, $\left(\mu_{B_{1}}^{U} \times \mu_{B_{2}}^{U}\right)\left(\left(x_{1}, x\right)\left(y_{1}, x\right)\right)=\left(\mu_{A_{1}}^{U} \times \mu_{A_{2}}^{U}\right)\left(x_{1}, x\right) \wedge\left(\mu_{A_{1}}^{U} \times \mu_{A_{2}}^{U}\right)\left(y_{1}, x\right)$. Ad-

$$
\begin{aligned}
\left(\sigma_{B_{1}} \times \sigma_{B_{2}}\right)\left(\left(x_{1}, x\right)\left(y_{1}, x\right)\right) & =\sigma_{B_{1}}\left(x_{1} y_{1}\right) \wedge \sigma_{A_{2}}(x) \\
& =\sigma_{A_{1}}\left(x_{1}\right) \wedge \sigma_{A_{1}}\left(y_{1}\right) \wedge \sigma_{A_{2}}(x) \\
& =\sigma_{A_{1}}\left(x_{1}\right) \wedge \sigma_{A_{2}}(x) \wedge \sigma_{A_{1}}\left(y_{1}\right) \wedge \sigma_{A_{2}}(x) \\
& =\left(\sigma_{A_{1}} \times \sigma_{A_{2}}\right)\left(x_{1}, x\right) \wedge\left(\sigma_{A_{1}} \times \sigma_{A_{2}}\right)\left(y_{1}, x\right) .
\end{aligned}
$$

This implies that the $\left(x_{1}, x\right)\left(y_{1}, x\right)$ is a CSE in $\mathcal{G}_{1} \times \mathcal{G}_{2}$. Since $x_{1} y_{1}$ is a CSE in $\mathcal{G}_{1}$, then, $x_{1} \in C_{1}$ or $y_{1} \in C_{1}$, therefore, the vertices $\left(x_{1}, x\right) \in C_{1} \times\left(V_{2}^{*} \backslash C_{2}\right)$ or $\left(y_{1}, x\right) \in C_{1} \times\left(V_{2}^{*} \backslash\right.$ $\left.C_{2}\right)$ in $\mathcal{G}_{1} \times \mathcal{G}_{2}$. Thus, the CSEs of this form are covered by the set $C_{1} \times\left(V_{2}^{*} \backslash C_{1}\right)$. Therefore, the set $\left\{C_{1} \times\left(V_{2}^{*} \backslash C_{2}\right)\right\} \cup\left\{\left(V_{1}^{*} \backslash C_{1}\right) \times C_{2}\right\}$ is covering all the CSEs in $\mathcal{G}_{1} \times \mathcal{G}_{2}$.

Example 8. Consider two CGs $\mathcal{G}_{1}$ and $\mathcal{G}_{2}$ in Example 6. The Cartesian product of $\mathcal{G}_{1}$ and $\mathcal{G}_{2}$ is given in Figure 7.

We have

$$
\begin{aligned}
& \left|C_{1} \times\left(V_{2}^{*} \backslash C_{2}\right)\right|=\left|\left\{x_{1}\right\} \times\left\{x_{2}\right\}=\right|\left\{x_{1} x_{2}\right\}=0.56, \\
& \left|\left(V_{1}^{*} \backslash C_{1}\right) \times C_{2}\right|=\left|\left\{y_{1}\right\} \times\left\{y_{2}\right\}\right|=\mid\left\{y_{1} y_{2}\right\}=0.53 .
\end{aligned}
$$

Therefore,

$$
\alpha_{s}\left(\mathcal{G}_{1} \times \mathcal{G}_{2}\right)=\left|\left\{C_{1} \times\left(V_{2}^{*} \backslash C_{2}\right)\right\} \cup\left\{\left(V_{1}^{*} \backslash C_{1}\right) \times C_{2}\right\}\right|=0.56+0.53=1.09 .
$$

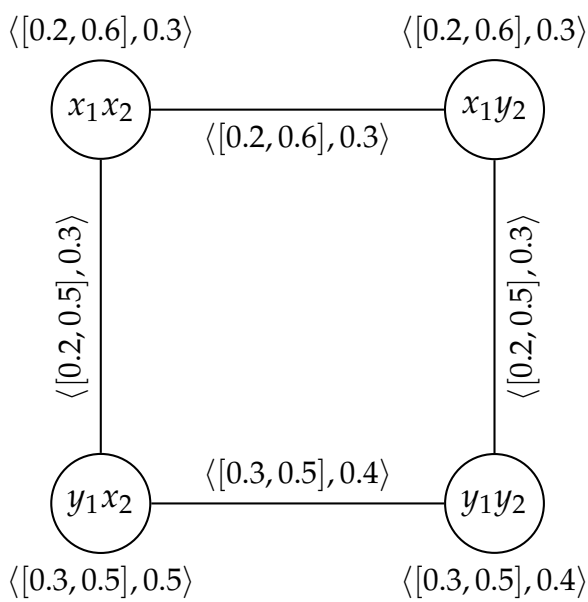

Figure 7. The Cartesian product $\mathcal{G}_{1}$ and $\mathcal{G}_{2}$. 
Corollary 8. Let $C_{1}$ and $C_{2}$ be the $\alpha_{s}$-sets of $\mathcal{G}_{1}$ and $\mathcal{G}_{2}$, respectively. If the $C G \mathcal{G}_{1} \times \mathcal{G}_{2}$ is the Cartesian product of $\mathcal{G}_{1}$ and $\mathcal{G}_{2}$, then,

$$
\alpha_{s}\left(\mathcal{G}_{1} \times \mathcal{G}_{2}\right)=\left|\left\{C_{1} \times\left(V_{2}^{*} \backslash C_{2}\right)\right\} \cup\left\{\left(V_{1}^{*} \backslash C_{1}\right) \times C_{2}\right\}\right| .
$$

At the end, the strong vertex covering number is determined in the composition of two cubic graphs.

Definition 18. Consider $\mathcal{G}_{1}=\left(A_{1}, B_{1}\right)$ and $\mathcal{G}_{2}=\left(A_{2}, B_{2}\right)$ as the two CGs of the underlying graphs $\mathcal{G}_{1}^{*}$ and $\mathcal{G}_{2}^{*}$, respectively. The composition of $\mathcal{G}_{1}$ and $\mathcal{G}_{2}$ is shown by $\mathcal{G}_{1} \circ \mathcal{G}_{2}=\left(A_{1} \circ\right.$ $\left.A_{2}, B_{1} \circ B_{2}\right)$ and is described as:

(i) $\left\{\begin{array}{l}{\left[\mu_{A_{1}}^{L} \circ \mu_{A_{2}}^{L} \mu_{A_{1}}^{U} \circ \mu_{A_{2}}^{U}\right]\left(x_{1}, x_{2}\right)=\left[\mu_{A_{1}}^{L}\left(x_{1}\right) \wedge \mu_{A_{2}}^{L}\left(x_{2}\right), \mu_{A_{1}}^{U}\left(x_{1}\right) \wedge \mu_{A_{2}}^{U}\left(x_{2}\right)\right],} \\ \left(\sigma_{A_{1}} \circ \sigma_{A_{2}}\right)\left(x_{1}, x_{2}\right)=\sigma_{A_{1}}\left(x_{1}\right) \wedge \sigma_{A_{2}}\left(x_{2}\right), \quad \forall\left(x_{1}, x_{2}\right) \in V_{1}^{*} \times V_{2}^{*} .\end{array}\right.$

(ii) $\left\{\begin{array}{l}{\left[\mu_{B_{1}}^{L} \circ \mu_{B_{2}}^{L}, \mu_{B_{1}}^{U} \circ \mu_{B_{2}}^{U}\right]\left(\left(z, x_{2}\right)\left(z, y_{2}\right)\right)=\left[\mu_{A_{1}}^{L}(z) \wedge \mu_{B_{2}}^{L}\left(x_{2} y_{2}\right), \mu_{A_{1}}^{U}(z) \wedge \mu_{B_{2}}^{U}\left(x_{2} y_{2}\right)\right],} \\ \left(\sigma_{B_{1}} \circ \sigma_{B_{2}}\right)\left(\left(z, x_{2}\right)\left(z, y_{2}\right)\right)=\sigma_{A_{1}}(z) \wedge \sigma_{B_{2}}\left(x_{2} y_{2}\right), \quad \forall z \in V_{1}^{*}, x_{2} y_{2} \in E_{2}^{*} .\end{array}\right.$

(iii) $\left\{\begin{array}{l}{\left[\mu_{B_{1}}^{L} \circ \mu_{B_{2}}^{L}, \mu_{B_{1}}^{U} \circ \mu_{B_{2}}^{U}\right]\left(\left(x_{1}, z\right)\left(y_{1}, z\right)\right)=\left[\mu_{B_{1}}^{L}\left(x_{1} y_{1}\right) \wedge \mu_{A_{2}}^{L}(z), \mu_{B_{1}}^{U}\left(x_{1} y_{1}\right) \wedge \mu_{A_{2}}^{U}(z)\right],} \\ \left(\sigma_{B_{1}} \circ \sigma_{B_{2}}\right)\left(\left(x_{1}, z\right)\left(y_{1}, z\right)\right)=\sigma_{B_{1}}\left(x_{1} y_{1}\right) \wedge \sigma_{A_{2}}(z), \quad \forall z \in V_{2}^{*}, x_{1} y_{1} \in E_{1}^{*} .\end{array}\right.$

(iv) $\left\{\begin{array}{l}{\left[\mu_{B_{1}}^{L} \circ \mu_{B_{2}}^{L}, \mu_{B_{1}}^{U} \circ \mu_{B_{2}}^{U}\right]\left(\left(x_{1}, x_{2}\right)\left(y_{1}, y_{2}\right)\right)} \\ =\left[\mu_{A_{1}}^{L}\left(x_{1}\right) \wedge \mu_{A_{1}}^{L}\left(y_{1}\right) \wedge \mu_{B_{2}}^{L}\left(x_{2} y_{2}\right), \mu_{A_{1}}^{U}\left(x_{1}\right) \wedge \mu_{A_{1}}^{U}\left(y_{1}\right) \wedge \mu_{B_{2}}^{U}\left(x_{2} y_{2}\right)\right], \\ \left(\sigma_{B_{1}} \circ \sigma_{B_{2}}\right)\left(\left(x_{1}, x_{2}\right)\left(y_{1}, y_{2}\right)\right)=\sigma_{A_{1}}\left(x_{1}\right) \wedge \sigma_{A_{1}}\left(y_{1}\right) \wedge \sigma_{B_{2}}\left(x_{2} y_{2}\right), \quad \forall x_{1} y_{1} \in E_{1}^{*}, x_{2} y_{2} \in E_{2}^{*} .\end{array}\right.$

Theorem 13. Consider $C_{1}$ and $C_{2}$ as the two SVCSs of the CGs $\mathcal{G}_{1}=\left(A_{1}, B_{1}\right)$ and $\mathcal{G}_{2}=\left(A_{2}, B_{2}\right)$, respectively. Then, $\left(C_{1} \times V_{2}^{*}\right) \cup\left(V_{1}^{*} \times C_{2}\right)$ is the SVCS of $\mathcal{G}_{1} \circ \mathcal{G}_{2}$.

Proof. Let $C_{1}$ and $C_{2}$ be the two SVCSs of the CGs $\mathcal{G}_{1}$ and $\mathcal{G}_{2}$, respectively.

Case (i). $z \in V_{1}^{*}$ and $x_{2} y_{2} \in E_{2}^{*}$.

If $x_{2} y_{2}$ is a CSE in $\mathcal{G}_{2}$, then,

$$
\begin{aligned}
\left(\mu_{B_{1}}^{L} \circ \mu_{B_{2}}^{L}\right)\left(\left(z, x_{2}\right)\left(z, y_{2}\right)\right) & =\mu_{A_{1}}^{L}(z) \wedge \mu_{B_{2}}^{L}\left(x_{2} y_{2}\right) \\
& =\mu_{A_{1}}^{L}(z) \wedge \mu_{A_{2}}^{L}\left(x_{2}\right) \wedge \mu_{A_{2}}^{L}\left(y_{2}\right) \\
& =\mu_{A_{1}}^{L}(z) \wedge \mu_{A_{2}}^{L}\left(x_{2}\right) \wedge \mu_{A_{1}}^{L}(z) \wedge \mu_{A_{2}}^{L}\left(y_{2}\right) \\
& =\left(\mu_{A_{1}}^{L} \circ \mu_{A_{2}}^{L}\right)\left(z, x_{2}\right) \wedge\left(\mu_{A_{1}}^{L} \circ \mu_{A_{2}}^{L}\right)\left(z, y_{2}\right) .
\end{aligned}
$$

Similarly, $\left(\mu_{B_{1}}^{U} \circ \mu_{B_{2}}^{U}\right)\left(\left(z, x_{2}\right)\left(z, y_{2}\right)\right)=\left(\mu_{A_{1}}^{U} \circ \mu_{A_{2}}^{U}\right)\left(z, x_{2}\right) \wedge\left(\mu_{A_{1}}^{U} \circ \mu_{A_{2}}^{U}\right)\left(z, y_{2}\right)$.

$$
\begin{aligned}
\left(\sigma_{B_{1}} \circ \sigma_{B_{2}}\right)\left(\left(z, x_{2}\right)\left(z, y_{2}\right)\right) & =\sigma_{A_{1}}(z) \wedge \sigma_{B_{2}}\left(x_{2} y_{2}\right) \\
& =\sigma_{A_{1}}(z) \wedge \sigma_{A_{2}}\left(x_{2}\right) \wedge \sigma_{A_{2}}\left(y_{2}\right) \\
& =\sigma_{A_{1}}(z) \wedge \sigma_{A_{2}}\left(x_{2}\right) \wedge \sigma_{A_{1}}(z) \wedge \sigma_{A_{2}}\left(y_{2}\right) \\
& =\left(\sigma_{A_{1}} \circ \sigma_{A_{2}}\right)\left(z, x_{2}\right) \wedge\left(\sigma_{A_{1}} \circ \sigma_{A_{2}}\right)\left(z, y_{2}\right) .
\end{aligned}
$$

Therefore, $\left(z, x_{2}\right)\left(z, y_{2}\right)$ is a CSE in $\mathcal{G}_{1} \circ \mathcal{G}_{2}$. Since $x_{2} y_{2}$ is a CSE in $G_{2}$, so $x_{2} \in C_{2}$ or $y_{2} \in C_{2}$, thus, the vertices $\left(z, x_{2}\right) \in V_{1}^{*} \times C_{2}$ or $\left(z, y_{2}\right) \in V_{1}^{*} \times C_{2}$ in $\mathcal{G}_{1} \times \mathcal{G}_{2}$. Then, $V_{1}^{*} \times C_{2}$ is an SVCS for these CSEs. 
Case (ii). $z \in V_{2}^{*}$ and $x_{1} y_{1} \in E_{1}^{*} \cdot x_{1} y_{1} \in E_{1}^{*}$ is a CSE, then,

$$
\begin{aligned}
\left(\mu_{B_{1}}^{L} \circ \mu_{B_{2}}^{L}\right)\left(\left(x_{1}, z\right)\left(y_{1}, z\right)\right) & =\mu_{B_{1}}^{L}\left(x_{1} y_{1}\right) \wedge \mu_{A_{2}}^{L}(z) \\
& =\mu_{A_{1}}^{L}\left(x_{1}\right) \wedge \mu_{A_{1}}^{L}\left(y_{1}\right) \wedge \mu_{A_{2}}^{L}(z) \\
& =\mu_{A_{1}}^{L}\left(x_{1}\right) \wedge \mu_{A_{2}}^{L}(z) \wedge \mu_{A_{1}}^{L}\left(y_{1}\right) \wedge \mu_{A_{2}}^{L}(z) \\
& =\left(\mu_{A_{1}}^{L} \circ \mu_{A_{2}}^{L}\right)(x, z) \wedge\left(\mu_{A_{1}}^{L} \circ \mu_{A_{2}}^{L}\right)\left(y_{1}, z\right) .
\end{aligned}
$$

Similarly,

$$
\begin{aligned}
\left(\mu_{B_{1}}^{U} \circ \mu_{B_{2}}^{U}\right)\left(\left(x_{1}, z\right)\left(y_{1}, z\right)\right) & =\left(\mu_{A_{1}}^{U} \circ \mu_{A_{2}}^{U}\right)(x, z) \wedge\left(\mu_{A_{1}}^{U} \circ \mu_{A_{2}}^{U}\right)\left(y_{1}, z\right), \\
\left(\sigma_{B_{1}} \circ \sigma_{B_{2}}\right)\left(\left(x_{1}, z\right)\left(y_{1}, z\right)\right) & =\left(\sigma_{A_{1}} \circ \sigma_{A_{2}}\right)\left(x_{1}, z\right) \wedge\left(\sigma_{A_{1}} \circ \sigma_{A_{2}}\right)\left(y_{1}, z\right) .
\end{aligned}
$$

Therefore $\left(x_{1}, z\right)\left(y_{1}, z\right)$ is a CSE in $\mathcal{G}_{1} \circ \mathcal{G}_{2}$, so that $x_{1} \in C_{1}$ or $y_{1} \in C_{1}$. Thus, $\left(x_{1}, z\right) \in$ $C_{1} \times V_{2}^{*}$ or $\left(y_{1}, z\right) \in C_{1} \times V_{2}^{*}$. Hence, $C_{1} \times V_{2}^{*}$ is the SVCS for these CSEs.

Case (iii). $x_{1} y_{1} \in E_{1}^{*}$ and $x_{2} y_{2} \in E_{2}^{*}$.

If $x_{1} y_{1} \in E_{1}^{*}$ and $x_{2} y_{2} \in E_{2}^{*}$ are the CSEs, then,

$$
\begin{aligned}
\left(\mu_{B_{1}}^{L} \circ \mu_{B_{2}}^{L}\right)\left(\left(x_{1}, x_{2}\right)\left(y_{1}, y_{2}\right)\right) & =\mu_{A_{1}}^{L}\left(x_{1}\right) \wedge \mu_{A_{1}}^{L}\left(y_{1}\right) \wedge \mu_{B_{2}}^{L}\left(x_{2} y_{2}\right) \\
& =\mu_{A_{1}}^{L}\left(x_{1}\right) \wedge \mu_{A_{1}}^{L}\left(y_{1}\right) \wedge \mu_{A_{2}}^{L}\left(x_{2}\right) \wedge \mu_{A_{2}}^{L}\left(y_{2}\right) \\
& =\left(\mu_{A_{1}}^{L} \circ \mu_{A_{2}}^{L}\right)\left(x_{1}, x_{2}\right) \wedge\left(\mu_{A_{1}}^{L} \circ \mu_{A_{2}}^{L}\right)\left(y_{1}, y_{2}\right) .
\end{aligned}
$$

Similarly,

$$
\begin{aligned}
\left(\mu_{B_{1}}^{U} \circ \mu_{B_{2}}^{U}\right)\left(\left(x_{1}, x_{2}\right)\left(y_{1}, y_{2}\right)\right) & =\left(\mu_{A_{1}}^{U} \circ \mu_{A_{2}}^{U}\right)\left(x_{1}, x_{2}\right) \wedge\left(\mu_{A_{1}}^{U} \circ \mu_{A_{2}}^{U}\right)\left(y_{1}, y_{2}\right), \\
\left(\sigma_{B_{1}} \circ \sigma_{B_{2}}\right)\left(\left(x_{1}, x_{2}\right)\left(y_{1}, y_{2}\right)\right) & =\left(\sigma_{A_{1}} \circ \sigma_{A_{2}}\right)\left(x_{1}, x_{2}\right) \wedge\left(\sigma_{A_{1}} \circ \sigma_{A_{2}}\right)\left(y_{1}, y_{2}\right) .
\end{aligned}
$$

Therefore, $\left(x_{1}, x_{2}\right)\left(y_{1}, y_{2}\right)$ is a CSE in $\mathcal{G}_{1} \circ \mathcal{G}_{2}$. Since $x_{2} \in C_{2}$ or $y_{2} \in C_{2}$, then, $\left(x_{1}, x_{2}\right) \in$ $V_{1}^{*} \times C_{2}$ or $\left(y_{1}, y_{2}\right) \in V_{1}^{*} \times C_{2}$. Thus, $\left(C_{1} \times V_{2}^{*}\right) \cup\left(V_{1}^{*} \times C_{2}\right)$ is the SVCS of CSEs in $\mathcal{G}_{1} \circ \mathcal{G}_{2}$.

Corollary 9. Consider $C_{1}$ and $C_{2}$ as the two $\alpha_{s}$-sets of $\mathcal{G}_{1}$ and $\mathcal{G}_{2}$, respectively. If the $C G \mathcal{G}_{1} \circ \mathcal{G}_{2}$ is the composition of $\mathcal{G}_{1}$ and $\mathcal{G}_{2}$, then,

$$
\alpha_{S}\left(\mathcal{G}_{1} \circ \mathcal{G}_{2}\right)=\left|\left(C_{1} \times V_{2}^{*}\right) \cup\left(V_{1}^{*} \times C_{2}\right)\right| .
$$

Example 9. The composition $\mathcal{G}_{1}$ and $\mathcal{G}_{2}$ in Example 6 is shown in Figure 8.

We have

$$
\begin{aligned}
& C_{1}=\left\{x_{1}\right\}, \quad C_{2}=\left\{y_{2}\right\}, \\
& V_{1}^{*} \times C_{2}=\left\{x_{1} y_{2}, y_{1} y_{2}\right\}, \quad C_{1} \times V_{2}^{*}=\left\{x_{1} x_{2}, x_{1} y_{2}\right\} .
\end{aligned}
$$

Therefore, $\left(V_{1}^{*} \times C_{2}\right) \cup\left(C_{1} \times V_{2}^{*}\right)=\left\{x_{1} x_{2}, x_{1} y_{2}, y_{1} y_{2}\right\}$.

$$
\alpha_{s}\left(\mathcal{G}_{1} \circ \mathcal{G}_{2}\right)=\left|\left\{x_{1} x_{2}, x_{1} y_{2}, y_{1} y_{2}\right\}\right|=0.56+0.56+0.53=1.65 \text {. }
$$




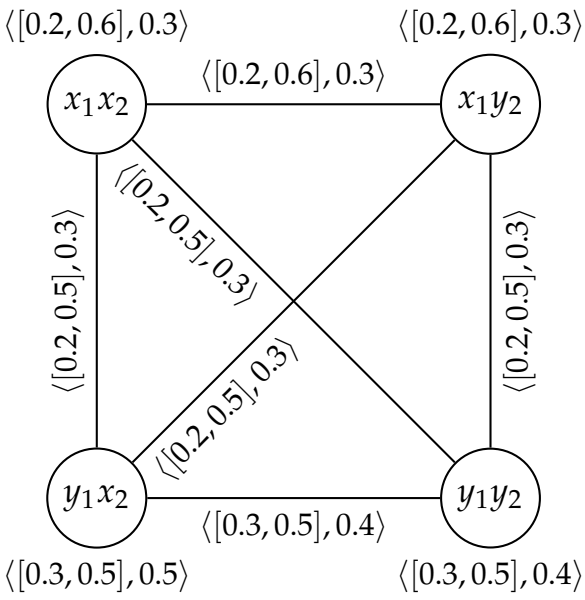

Figure 8. The composition of $\mathcal{G}_{1}$ and $\mathcal{G}_{2}$.

\section{Application}

\subsection{The Application of Strong Vertex Covering in NGA Monitoring Stations}

The Global Positioning System (GPS) is a system for locating geographical location. This system consists of 24 satellites that orbit the Earth and there are 4 satellites in each orbit. Control and space units were established and developed by the United States Air Force (USAF) and continue to operate today. Waves emitted from space are received by GPS satellites and by GPS receivers. These receivers are available to a variety of users and are used to calculate the three-dimensional location (latitude and longitude) of the desired location and time. All ground facilities necessary to support the GPS constellation are included within the control system, as shown in Figure 9.

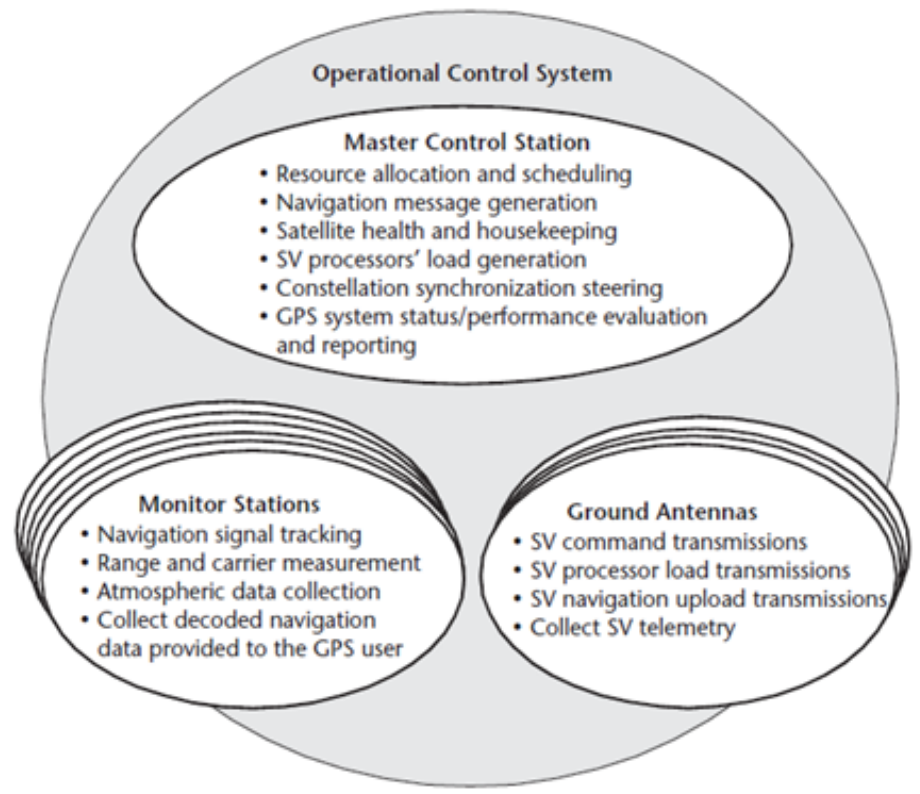

Figure 9. Control system overview. (Understanding GPS: principles and applications, Elliott Kaplan, Christopher Hegarty—2nd ed., 2006).

The National Geospatial Intelligence Agency (NGA) is a globally distributed network whose primary mission is to collect observations from the constellation GPS. They are spread out around the world and include atomic clock standards and GPS receivers for the continuous collection of GPS data for all satellites viewed from their locations. The collected data are sent to the main control station, where they are processed for estimating 
satellite circuits and clock errors, among other parameters, and to generate a navigation message. They also gather navigation signals, measurement ranges and atmospheric data.

Prior to the renovation program, the monitoring station network consisted of five sites including Hawaii, Colorado Springs (Colorado, USA), Ascension Island (South Atlantic), Diego Garcia (Indian Ocean), and Kwajalein (North Pacific).

To increase efficiency and accuracy, new stations were created on Earth that provided a larger view of the constellation, including Cape Canaveral (Florida, USA) in 2001 and 6 new stations in 2005, including Adelaide (Australia), Buenos Aires (Argentina), Hermitage (UK), Manama (Bahrain), Quito (Ecuador), and Washington DC (USA).

Five more stations were added in 2006, including Fairbanks (Alaska, USA), Papeete (Tahiti), Osan (South Korea), Pretoria (South Africa), and Wellington (New Zealand).

The network currently consists of 16 stations, of which 6 are from the Air Force plus 10 from the NGA. With this configuration, each satellite can be seen from at least three monitoring stations, which allows it to calculate more accurate orbits and transient data, thus improving system accuracy. The location of these stations is shown in Figure 10.

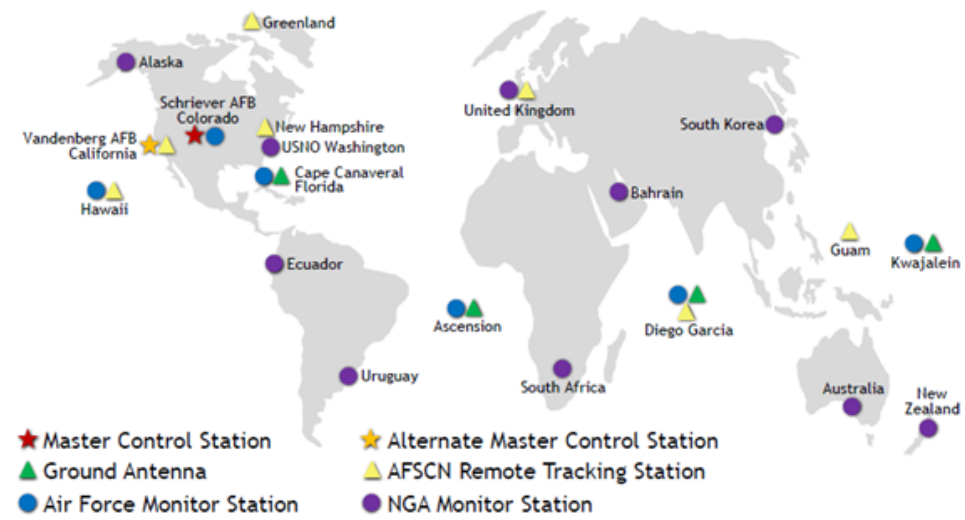

Figure 10. GPS control segment (https: / www.gps.gov/multimedia/images/GPS-control-segmentmap.pdf (accessed on 12 January 2022), Updated May 2017).

Since the Air Force monitoring station is one of the first monitoring stations created in the GPS system, it is essential that NGA monitoring stations communicate with them to exchange experiences and data. Because of the scatter and distance of the NGA monitoring station from the Air Force monitoring station, each NGA monitoring station can only be strongly connected to one or two of the nearest Air Force monitoring stations. This connection varies depending on the data sending and receiving power and the antenna power of each station. These variables are associated with uncertainty about time and place conditions. We show these values with fuzzy numbers. Since the power to send data is usually less than the power to receive them, it can be represented by an interval-valued fuzzy number. Therefore, each monitoring station power can be expressed by a cubic fuzzy number, in which interval-valued fuzzy membership is an interval of the power to send and receive data, and fuzzy membership is the power of the antenna. Similarly, the relationship between two stations can also be shown with a cubic fuzzy number in which the membership of the power to send and receive data between the two stations and its fuzzy membership is the number of antennae relative to each other. Therefore, by considering the monitoring stations as the vertices of a graph, we are dealing with a CG. We show the CG with the vertices of the monitoring stations in Figure 11. 


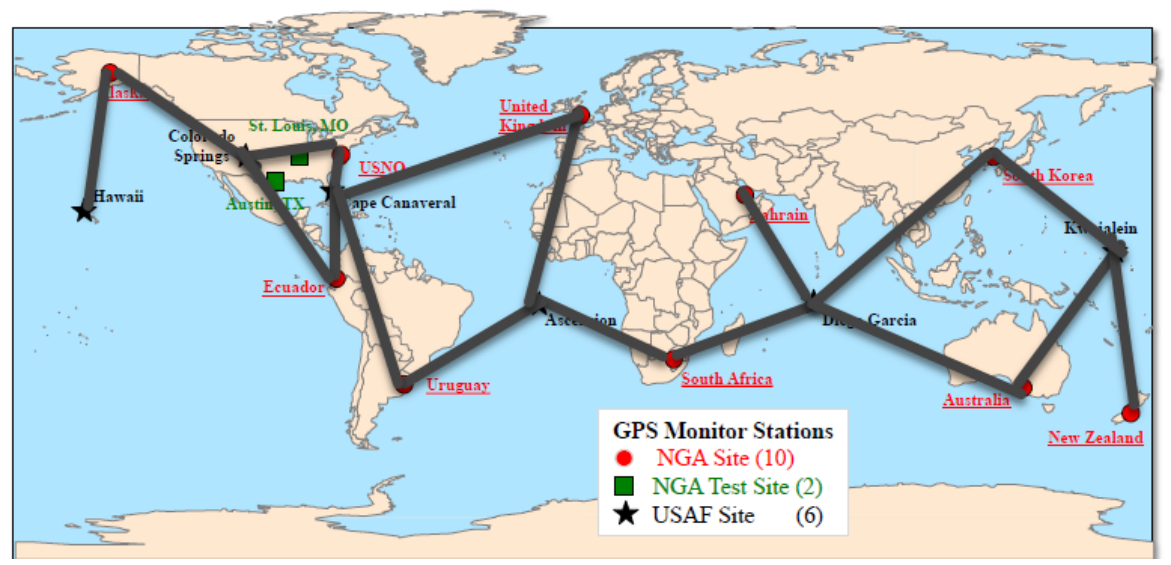

Figure 11. the CG with the vertices of the monitor stations. (Presentation to Civil GPS Service Interface Committee at ION GNSS, 2016, https:/ / www.gps.gov/cgsic/meetings/2016/wong.pdf (accessed on 12 January 2022), Approved for Public Release, 16-603).

It seems necessary to have stations for the monitoring station centralization and support. Therefore, in this graph, we are looking for a set of stations that strongly cover the connection between other stations. The cubic membership values of monitoring stations and the connection between them are shown in Tables 2 and 3.

Table 2. Cubic membership values of monitoring stations.

\begin{tabular}{cccccc}
\hline Vertex & Station & Membership & Vertex & Station & Membership \\
\hline A1 & Hawaii & $\langle[0.7,0.8], 0.8\rangle$ & A9 & United Kingdom & $\langle[0.6,0.7], 0.5\rangle$ \\
A2 & Alaska & $\langle[0.6,0.7], 0.7\rangle$ & A10 & South Africa & $\langle[0.7,0.8], 0.6\rangle$ \\
A3 & Colorado & $\langle[0.8,0.9], 0.9\rangle$ & A11 & Bahrain & $\langle[0.5,0.6], 0.6\rangle$ \\
A4 & USNO Washington & $\langle[0.8,0.9], 0.8\rangle$ & A12 & Diego Garcia & $\langle[0.7,0.9], 0.7\rangle$ \\
A5 & Cape Canaveral & $\langle[0.8,0.9], 0.9\rangle$ & A13 & South Korea & $\langle[0.6,0.7], 0.7\rangle$ \\
A6 & Ecuador & $\langle[0.7,0.8], 0.7\rangle$ & A14 & Kwajalein & $\langle[0.7,0.8], 0.8\rangle$ \\
A7 & Uruguay & $\langle[0.5,0.7], 0.6\rangle$ & A15 & Australia & $\langle[0.6,0.8], 0.7\rangle$ \\
A8 & Ascension & $\langle[0.7,0.8], 0.7\rangle$ & A16 & New Zealand & $\langle[0.6,0.7], 0.6\rangle$ \\
\hline
\end{tabular}

Table 3. Cubic membership values among monitoring stations.

\begin{tabular}{cccc}
\hline Hawaii-Alaska & $\langle[0.6,0.7], 0.7\rangle$ & Uruguay-Ascension & $\langle[0.5,0.7], 0.6\rangle$ \\
Alaska- Colorado & $\langle[0.6,0.7], 0.7\rangle$ & Ascension-United Kingdom & $\langle[0.6,0.7], 0.5\rangle$ \\
Colorado-USNO & $\langle[0.8,0.9], 0.8\rangle$ & Ascension-South Africa & $\langle[0.7,0.8], 0.6\rangle$ \\
USNO- Cape Canaveral & $\langle[0.8,0.9], 0.8\rangle$ & South Africa-Diego Garcia & $\langle[0.7,0.8], 0.6\rangle$ \\
Colorado-Ecuador & $\langle[0.7,0.8], 0.7\rangle$ & Bahrain- Diego Garcia & $\langle[0.5,0.6], 0.6\rangle$ \\
Cape Canaveral-Ecuador & $\langle[0.7,0.8], 0.7\rangle$ & Diego Garcia-South Korea & $\langle[0.6,0.7], 0.7\rangle$ \\
Cape Canaveral-Uruguay & $\langle[0.5,0.7], 0.6\rangle$ & Diego Garcia-Australia & $\langle[0.6,0.8], 0.7\rangle$ \\
Cape Canaveral-United & $\langle[0.6,0.7], 0.5\rangle$ & South Korea-Kwajalein & $\langle[0.6,0.7], 0.7\rangle$ \\
Kingdom & $\langle[0.6,0.8], 0.7\rangle$ & Kwajalein-New Zealand & $\langle[0.6,0.7], 0.6\rangle$ \\
Kwajalein-Australia & & &
\end{tabular}

The minimal SVCSs are as follows:

$$
\begin{aligned}
& C_{1}=\{A 2, A 3, A 5, A 8, A 12, A 14\}, \\
& C_{2}=\{A 1, A 3, A 5, A 8, A 12, A 14\}, \\
& C_{3}=\{A 2, A 4, A 6, A 7, A 9, A 10, A 12, A 14\}, \\
& C_{4}=\{A 1, A 3, A 5, A 7, A 9, A 10, A 12, A 14\}, \\
& C_{5}=\{A 1, A 3, A 5, A 8, A 12, A 13, A 15, A 16\}, \\
& C_{6}=\{A 2, A 4, A 6, A 7, A 9, A 10, A 11, A 13, A 15, A 16\} .
\end{aligned}
$$


By calculating the above cardinality of SVCSs, we have

$$
\begin{aligned}
& \left|C_{1}\right|=3.78 \text {, } \\
& \left|C_{2}\right|=3.81 \text {, } \\
& \left|C_{3}\right|=4.78, \\
& \left|C_{4}\right|=4.9 \text {, } \\
& \left|C_{5}\right|=4.97 \text {, } \\
& \left|C_{6}\right|=5.87 \text {. }
\end{aligned}
$$

Therefore, $C_{1}$ is $\alpha_{s}$-set.

Examining the vertices of this set, we find that the minimum monitoring stations to support other monitoring stations are almost the same as the Air Force monitoring stations. In fact, the creation of new monitoring stations may have been covered by Air Force surveillance stations. If so, such stations should be established elsewhere. With this assumption, the creation of monitoring stations in the Far East and the Mediterranean basin does not seem far-fetched. As a result of the concentration of the surrounding countries, the existence of such a monitoring station is felt, especially in the Mediterranean Sea.

\subsection{The Application of Strong Vertex Independence in the Distribution of Facilities in Municipal Services}

One of the most important consequences of the rapid growth of urbanization and physical development of cities in recent decades has been the disintegration of the distribution system of service centers in the city, which has led to the social inequality of citizens in accessing these services. Today, the problems, caused by the inadequate distribution of urban services, have made the distribution of urban services one of the most important issues facing most developed and developing countries. In the last half-century, with the increase in urban population, more and more urban officials have been concerned with the provision of urban services, and less attention has been paid to the proper distribution of services. The unfair inter-city and intra-city distribution of facilities and resources has led to migration, social crises and complex spatial problems, increasing suburban travel and failing to meet the needs of citizens. Governorships and municipalities play an important role in distributing these facilities. Increasing the number of municipalities can be effective in expanding municipal services. It is clear that as the cities grow larger and more populous, they demand more services and facilities. One of the important factors in the distribution of facilities in a city is population density. Population growth as well as increased migration to cities in recent decades have led to an increase in sparsely populated areas. The higher the population density in a city, the more the need for facilities to be distributed. Population growth rate is another important factor that influences the decisions of city officials.

Using a CG can be useful in modeling urban issues in relation to the population density. CG can also be useful for city officials, as it helps the governor and mayor make appropriate policies for the city to develop appropriate services. Since the power of governorships and municipalities and population density in cities are uncertain values, we are faced with fuzzy numbers. Table 4 shows the information from 12 cities in Mazandaran province in Iran in terms of population density and number of municipal areas. (https://www.amar.org.ir/Portals/0/census/1385/results/kolli/ostan02.rar (accessed on 12 January 2022), 2007).

The location of these cities is shown in Figure 12.

Urban population density and the power of city officials in the distribution of urban services are calculated in Table 5. Fuzzy values for the power of city officials is calculated as the ratio of the number of municipal areas in each city to a unit greater than the maximum number of municipal areas in Table 4. Since the population density varies based on natural disasters, diseases, migration, and births, it can be represented by an interval-valued fuzzy number. In calculating the interval-valued membership, the following formulas are used, in which $u d_{i}, r$ and $r_{\max }$ are the urban density, the population growth rate of the city and the growth rate of the city with the maximum value, respectively.

$$
m_{i}=\frac{u d_{i}}{\max \left(u d_{i}\right)+1}, \quad m_{i}^{\prime}=\frac{u d_{i} \times r_{i}}{\max \left(u d_{i}\right) \times r_{\max }+1}, \quad h=\left|m_{i}^{\prime}-m_{i}\right| .
$$


In this case, the interval-valued membership is equal to $\left[m_{i}-h, m_{i}+h\right]$.

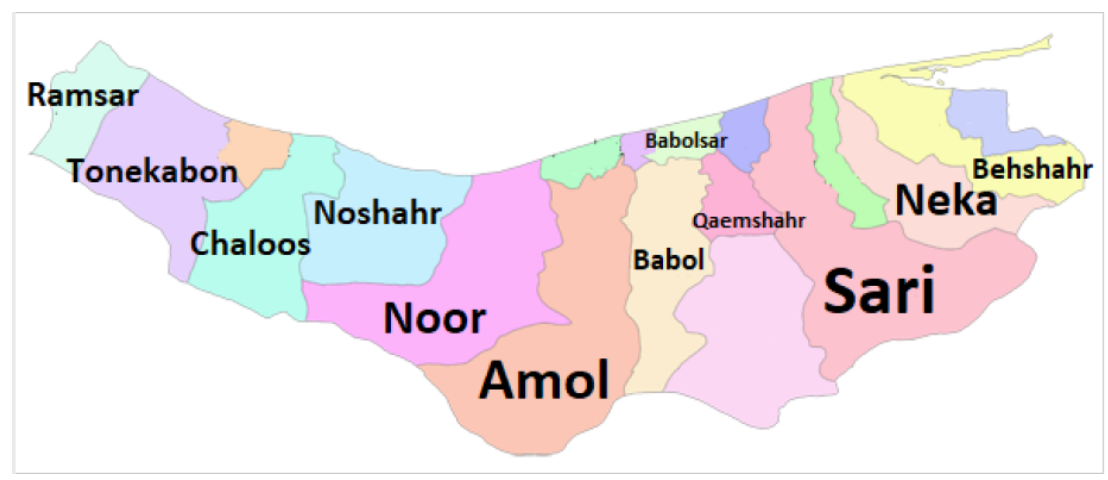

Figure 12. Map of cities.

Table 4. Related datasets to the cities.

\begin{tabular}{|c|c|c|c|c|c|c|c|}
\hline Cities & $\begin{array}{c}\text { Area } \\
\left(\mathrm{km}^{2}\right)\end{array}$ & $\begin{array}{c}\text { Urban } \\
\text { Population }\end{array}$ & $\begin{array}{l}\text { Urban } \\
\text { Density }\end{array}$ & $\begin{array}{l}\text { Suburban } \\
\text { Population }\end{array}$ & $\begin{array}{l}\text { Suburban } \\
\text { Density }\end{array}$ & Growth Rate & $\begin{array}{c}\text { Governorships } \\
\text { and Municipal } \\
\text { Areas }\end{array}$ \\
\hline Amol & 3074.4 & 199,698 & 65 & 202,369 & 113 & 1.38 & 4 \\
\hline Babol & 1578.1 & 201,335 & 127 & 240,073 & 297 & 1.10 & 4 \\
\hline Babolsar & 246.5 & 50,032 & 203 & 94,937 & 385 & 1.22 & 2 \\
\hline Behshahr & 1416.3 & 84,117 & 59 & 105,651 & 110 & 1.20 & 3 \\
\hline Chaloos & 1089.5 & 45,625 & 28 & 64,726 & 77 & 1.12 & 2 \\
\hline Neka & 1358.8 & 46,291 & 77 & 46,291 & 34 & 0.96 & 2 \\
\hline Noor & 2675 & 46,569 & 9 & 22,491 & 39 & 1.12 & 4 \\
\hline Noshahr & 1716.5 & 42,175 & 69 & 42,175 & 24 & 1.08 & 2 \\
\hline Qaemshahr & 371 & 174,768 & 643 & 182,240 & 381 & 1.21 & 4 \\
\hline Ramsar & 729.8 & 32,085 & 34 & 50,040 & 93 & 0.56 & 2 \\
\hline Sari & 3248.4 & 261,293 & 134 & 273,972 & 71 & 1.53 & 5 \\
\hline Tonekabon & 1732.2 & 43,842 & 22 & 86,153 & 95 & 1.13 & 2 \\
\hline
\end{tabular}

Table 5. Outlining calculations.

\begin{tabular}{ccccccc}
\hline Cities & $\boldsymbol{i}$ & $\boldsymbol{u d}_{\boldsymbol{i}}$ & $\boldsymbol{m}_{\boldsymbol{i}}$ & $\boldsymbol{r}_{\boldsymbol{i}}$ & $\boldsymbol{m}_{\boldsymbol{i}}^{\prime}$ & $\boldsymbol{h}$ \\
\hline Amol & 1 & 65 & 0.101 & 1.38 & 0.115 & 0.014 \\
Babol & 2 & 127 & 0.197 & 1.10 & 0.179 & 0.018 \\
Babolsar & 3 & 203 & 0.315 & 1.22 & 0.318 & 0.003 \\
Behshahr & 4 & 59 & 0.0916 & 1.20 & 0.0909 & 0.0007 \\
Chaloos & 5 & 28 & 0.043 & 1.12 & 0.040 & 0.003 \\
Neka & 6 & 77 & 0.119 & 0.96 & 0.095 & 0.024 \\
Noor & 7 & 9 & 0.014 & 1.12 & 0.013 & 0.001 \\
Noshahr & 8 & 69 & 0.107 & 1.08 & 0.096 & 0.011 \\
Qaemshahr & 9 & 643 & 0.998 & 1.21 & 0.999 & 0.001 \\
Ramsar & 10 & 34 & 0.052 & 0.056 & 0.024 & 0.028 \\
Sari & 11 & 134 & 0.208 & 1.53 & 0.263 & 0.055 \\
Tonekabon & 12 & 22 & 0.034 & 1.13 & 0.032 & 0.002 \\
\hline
\end{tabular}

The cubic membership degrees of the cities are shown in Table 6. 
Table 6. Cubic values and cardinal of cities.

\begin{tabular}{ccc}
\hline Cities & Cubic Values & Cardinal of Cities \\
\hline Amol & $\langle[0.087,0.115], 0.66\rangle$ & 0.562 \\
Babol & $\langle[0.179,0.215], 0.66\rangle$ & 0.565 \\
Babolsar & $\langle[0.312,0.318], 0.33\rangle$ & 0.445 \\
Behshahr & $\langle[0.091,0.092], 0.50\rangle$ & 0.500 \\
Chaloos & $\langle[0.046,0.083], 0.33\rangle$ & 0.455 \\
Neka & $\langle[0.095,0.143], 0.33\rangle$ & 0.459 \\
Noor & $\langle[0.013,0.015], 0.66\rangle$ & 0.554 \\
Noshahr & $\langle[0.096,0.118], 0.33\rangle$ & 0.450 \\
Qaemshahr & $\langle[0.997,0.999], 0.66\rangle$ & 0.554 \\
Ramsar & $\langle[0.024,0.080], 0.33\rangle$ & 0.462 \\
Sari & $\langle[0.153,0.263], 0.83\rangle$ & 0.646 \\
Tonekabon & $\langle[0.032,0.036], 0.33\rangle$ & 0.444 \\
\hline
\end{tabular}

Distribution of facilities in cities can affect neighboring cities. People travel there to solve some of their problems if there are better centers in nearby cities. City officials and municipalities have also built some facilities in inter-city areas so that they can provide proper services to people between cities as well as travelers. Communication between cities is usually possible from the main road and some side roads. As a result of higher congestion and traveling, main roads have more facilities than the side roads. The connection of cities through the main road is considered a strong connection between the two cities. Cubic values between cities are shown in Table 7 and its CG in Figure 13.

Table 7. Cubic values between cities.

\begin{tabular}{cc}
\hline Main Roads between Cities & Cubic Values \\
\hline Babolsar-Qaemshahr & $\langle[0.312,0.318], 0.33\rangle$ \\
Sari-Qaemshahr & $\langle[0.153,0.263], 0.66\rangle$ \\
Sari- Neka & $\langle[0.095,0.143], 0.33\rangle$ \\
Neka-Behshahr & $\langle[0.091,0.092], 0.33\rangle$ \\
Babol-Amol & $\langle[0.087,0.115], 0.66\rangle$ \\
Babol-Babolsar & $\langle[0.179,0.215], 0.33\rangle$ \\
Babol-Qaemshahr & $\langle[0.179,0.215], 0.66\rangle$ \\
Amol-Noor & $\langle[0.013,0.015], 0.66\rangle$ \\
Nooor-Noshahr & $\langle[0.013,0.015], 0.33\rangle$ \\
Tonekabon-Ramsar & $\langle[0.024,0.036], 0.33\rangle$ \\
Chaloos-Noshahr & $\langle[0.046,0.083], 0.33\rangle$ \\
Chaloos-Tonekabon & $\langle[0.032,0.036], 0.33\rangle$ \\
Babolsar-Noor & $\langle[0.013,0.015], 0.33\rangle$ \\
Babolsar-Amol & $\langle[0.087,0.115], 0.33\rangle$ \\
Babolsar-Sari & $\langle[0.153,0.263], 0.33\rangle$ \\
\hline
\end{tabular}




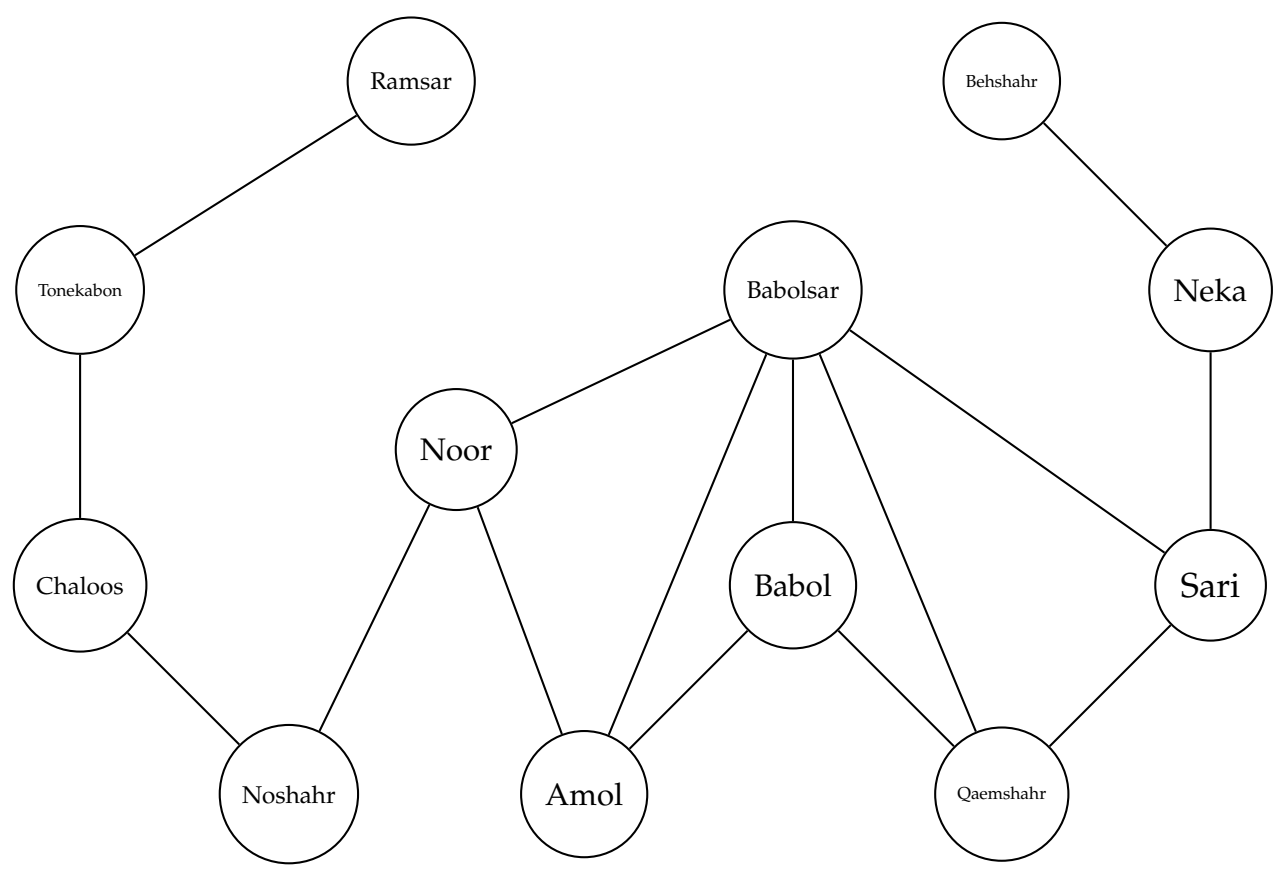

Figure 13. CG of cities.

In general, there is no polynomial algorithm for finding a maximum independent set for an arbitrary graph. This means that it is not possible to access such a collection in a short time. To obtain the maximum SVIS in CG with a small number of vertices, we used Sage [44] software according to the following instructions, and the results are shown in Table 8.

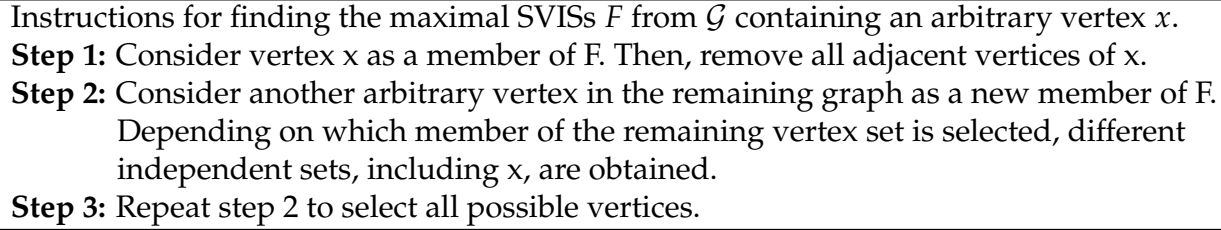

Instructions for finding the maximal SVISs $F$ from $\mathcal{G}$ containing an arbitrary vertex $x$

Step 1: Consider vertex $x$ as a member of $F$. Then, remove all adjacent vertices of $x$.

Step 2: Consider another arbitrary vertex in the remaining graph as a new member of $F$. Depending on which member of the remaining vertex set is selected, different independent sets, including $x$, are obtained.

Step 3: Repeat step 2 to select all possible vertices.

Therefore, it is concluded that $\{$ Chaloos, Babol, Noor, Ramsar, Sari, Behshahr $\}$ is the maximum SVIS with cardinal 3.182 and the cities mentioned in this set are completely independent of each other, which means that the necessary facilities and equipment for the citizens of these cities are fully available, and people do not need to migrate to other cities to make their living. This can greatly affect the important factor of population density in other cities. Therefore, governments should annually allocate the necessary funds to the officials of each city to create services and welfare facilities so that by creating these centers, the people of that city can easily benefit from these services without having to travel to distant cities for medical and welfare issues. This saves time and money for city-dwellers and increases the number of independent cities, which can be a very important factor in the fair distribution of urban facilities. 
Table 8. Calculation of SVISs by Sage software.

\begin{tabular}{|c|c|}
\hline SVISs & Cardinals \\
\hline ['Amol',' Chaloos',' Qaemshahr',' Ramsar','Neka'] & 2.492 \\
\hline ['Amol',' Chaloos',', Qaemshahr',', Ramsar',' Behshahr'] & 2.533 \\
\hline ['Amol',' Chaloos',' Ramsar','Sari','Behshahr'] & 2.625 \\
\hline ['Amol', Tonekabon', Qaemshahr','Noshahr','Neka'] & 2.469 \\
\hline ['Amol','Tonekabon',' Qaemshahr',' Noshahr',' Behshahr'] & 2.51 \\
\hline ['Amol',' Tonekabon',' Noshahr','Sari','Behshahr'] & 2.602 \\
\hline ['Amol',' Qaemshahr',' Ramsar',',Noshahr','Neka'] & 2.459 \\
\hline ['Amol',' Q Qaemshahr',' Ramsar',' Noshahr',' Behshahr'] & 2.528 \\
\hline$\left[{ }^{\prime}\right.$ Amol' $^{\prime},{ }^{\prime}$ Ramsar',' Noshahr','Sari','Behshahr'] & 2.62 \\
\hline$\left[{ }^{\prime}\right.$ Chaloos',' Babolsar',', Ramsar',', Neka'] & 1.821 \\
\hline ['Chaloos',' Babolsar',' Ramsar',' Behshahr'] & 1.862 \\
\hline 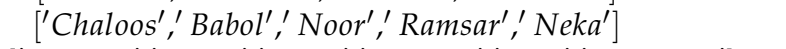 & 2.459 \\
\hline$\left[{ }^{\prime}\right.$ Chaloos',' Babol',' Noor',', Ramsar','Sari', Behshahr'] & 3.182 \\
\hline$\left[{ }^{\prime}\right.$ Chaloos',' Qaemshahr',' Noor',' Ramsar',' Neka'] & 2.484 \\
\hline ['Chaloos',' Qaemshahr','Noor','Ramsar',' Behshahr'] & 2.525 \\
\hline ['Babolsar','Tonekabon',' Noshahr',' Neka'] & 1.798 \\
\hline ['Babolsar','Tonekabon',' Noshahr',' Behshahr'] & 1.839 \\
\hline ['Babolsar',' Ramsar',' Noshahr',', Neka'] & 1.816 \\
\hline ['Babolsar','Ramsar',' Noshahr','Behshahr'] & 1.857 \\
\hline$\left[{ }^{\prime}\right.$ Tonekabon','Babol',',Noor',',Neka'] & 2.022 \\
\hline ['Tonekabon','Babol','Noor','Sari','Behshahr'] & 2.709 \\
\hline ['Tonekabon','Babol','Noshahr',',Neka'] & 1.918 \\
\hline$\left[{ }^{\prime}\right.$ Tonekabon','Babol',' Noshahr',' Sari','Behshahr' & 2.605 \\
\hline ['Tonekabon',' Qaemshahr','Noor',',Neka'] & 2.011 \\
\hline ['Tonekabon',' Qaemshahr','Noor','Behshahr'] & 2.052 \\
\hline ['Babol',' Ramsar','Noshahr','Neka'] & 1.936 \\
\hline ['Babol',' Ramsar',' Noshahr',' Sari',' Behshahr'] & 2.623 \\
\hline
\end{tabular}

\section{Conclusions}

Graph theory is a modeling tool for solving various problems in areas such as networking, connectivity, data analysis, cluster analysis, signal processing, image optimization, and scheduling, in which VC has special importance and applications. The VC is a fundamental issue in fuzzy graph theory that has wide applications in the real world. In other words, it can be stated that the VC of a graph is the subset of vertices in a graph that has at least one end point of every edge. The FG is one of the powerful tools in modeling uncertain phenomena. When it is not possible to model uncertainty problems with FGs and IVFGs, the CGs, as a combination, are a good option for solving these problems. The limitation of previous definitions in this field have led us to introduce the VC in a CG. In this study, we introduced and determined the SVCS number and the SVIS number in a CG by CSEs. We obtained these values on some specific CGs. This study showed that in SVCS, the minimum number of vertices, and in SVIS, the maximum number of vertices are valuable. This concept was studied on cube graphs with an omitted vertex. The results revealed that the omission of a vertex seriously disrupted the vertex covering. The boundaries obtained for SVCS indicated that the SVCSs number should not be less than the minimum degree of vertices of a CG. Then, the relationship between SVCS and SVIS was determined and the SVCS number on the most important CG operations was studied. Finally, the results of the SVCS and SVIS numbers in the CG were used to determine the NGA monitoring stations to link to each other and the distribution of facilities in municipal services, respectively. In future work, we will define the domination of the cubic graphs in terms of strong edges and examine their properties. Likewise, we will investigate domination in terms of independent sets and, since many of the phenomena surrounding us are hybrid, we will also discuss the domination concept regarding its fuzzy operations. 


\begin{abstract}
Author Contributions: H.J., A.A.T., Z.S. and S.H.S.; methodology, H.J., A.A.T., Z.S. and H.R.; validation, A.A.T., Z.S. and H.R.; formal analysis, H.J., Z.S. and A.A.T.; investigation, H.J., A.A.T., Z.S., S.H.S. and H.R.; data curation, H.J., A.A.T., Z.S. and S.H.S.; writing-original draft preparation, H.J. and A.A.T.; writing一review and editing, H.J., A.A.T., Z.S, H.R. and S.H.S.; visualization, H.J., A.A.T., H.R. and Z.S.; supervision, H.J. and Z.S.; project administration, H.J., A.A.T., Z.S., H.R. and S.H.S.; funding acquisition, Z.S. All authors have read and agreed to the published version of the manuscript.
\end{abstract}

Funding: This research received no external funding.

Institutional Review Board Statement: Not applicable.

Informed Consent Statement: Not applicable.

Data Availability Statement: Not applicable.

Conflicts of Interest: The authors declare no conflict of interest.

\title{
References
}

1. Håstad, J. Some optimal inapproximability results. J. ACM (JACM) 2001, 48, 798-859.

2. Dinur, I.; Safra, S. On the hardness of approximating minimum vertex cover. Ann. Math. 2005, 162, 439-485.

3. Ni, Y. Models, algorithm for stochastic minimum weight edge covering problem. In Proceedings of the Fourth International Conference on Information and Management Sciences, Yunnan, China, 26-28 August 2005; pp. 445-451.

4. Zadeh, L.A. Fuzzy sets. Inf. Control 1965, 8, 338-353.

5. Rosenfeld, A. Fuzzy Graphs, in Fuzzy Sets and Their Application to Cognitive and Decision Processes; Zadeh, L.A., Fu, K.S., Shimura, M., Eds.; Academic Press: New York, NY, USA, 1975; pp. 77-95.

6. $\quad$ Bhutani, K.R.; Rosenfeld, A. Strong arcs in fuzzy graphs. Inf. Sci. 2003, 152, 319-322.

7. Bhattacharya, P. Some remarks on fuzzy graphs. Pattern Recognit. Lett. 1987, 6, 297-302.

8. Mordeson, J.N.; Chang-Shyh, P. Operations on fuzzy graphs. Inf. Sci. 1994, 79, 159-170.

9. Akram, M.; Dudek, W.A. Interval-valued fuzzy graphs. Comput. Math. Appl. 2011, 61, 289-299.

10. Atanassov, K.T. Intuitionistic fuzzy sets. Fuzzy Sets Syst. 1986, 20, 87-96.

11. Rashmanlou, H.; Samanta, S.; Pal, M.; Borzooei, R.A. Intuitionistic fuzzy graphs with categorical properties. Fuzzy Inf. Eng. 2015, 7,317-334.

12. Dettlaff, M.; Lemanska, M.; Kosari, S.; Sheikholeslami, S. The convex domination subdivision number of a graph. Commun. Comb. Optim. 2016, 1, 43-56.

13. Kosari, S.; Rao, Y.; Jiang, H.; Liu, X.; Wu, P.; Shao, Z. Vague Graph Structure with Application in Medical Diagnosis. Symmetry 2020, 12, 1582

14. Rao, Y.; Kosari, S.; Shao, Z. Certain Properties of vague Graphs with a novel application. Mathematics 2020, 8, 1647.

15. Rao, Y.; Kosari, S.; Shao, Z.; Cai, R.; Xinyue, L. A Study on Domination in vague incidence graph and its application in medical sciences. Symmetry 2020, 12, 1885.

16. Shaebani, S.; Kosari, S.; Asgharsharghi, L. The restrained K-rainbow reinforcement number of graphs, Discrete Mathematics. Algorithms Appl. 2021, 13, 2150026.

17. Shi, X.; Kosari, S. Certain Properties of Domination in Product Vague Graphs with an Application in Medicine. Front. Phys. 2021, 9,680634 .

18. Shao, Z.; Kosari, S.; Rashmanlou, H.; Shoaib, M. New concepts in intuitionistic fuzzy graph with application in water supplier systems. Mathematics 2020, 8, 1241.

19. Talebi, A.A. Cayley fuzzy graphs on the fuzzy group. Comput. Appl. Math. 2018, 37, 4611-4632.

20. Talebi, A.A.; Rashmanlou, H.; Sadati, S.H. New concepts of m-polar interval valued intuitionistic fuzzy graph. TWMS J. Appl. Eng. Math. 2020, 10, 808-816.

21. Talebi, A.A.; Rashmanlou, H.; Sadati, S.H. Interval-valued Intuitionistic Fuzzy Competition Graph. J. Multi.-Valued Log. Soft Comput. 2020, 34, 335-364.

22. Akram, M.; Sitara, M. Certain fuzzy graph structures. J. Appl. Math. Comput. 2006, 61, 25-56.

23. Akram, M.; Sitara, M. Certain concepts in Intuitionistic Neutrosophic Graph Structures. Information 2017, 8, 154. doi:10.3390/info8040154.

24. Akram, M.; Sitara, M.; Saeid, A.B. Residue product of fuzzy graph structures. J. Mult.-Valued Log. Soft Comput. 2020, 34, 365-399.

25. Dinesh, T. A Study on Graph Structures, Incidence Algebras and Their Fuzzy Analogues. Ph.D. Thesis, Department of Mathematical Sciences, Kannur University Mangattuparamba Campus, Mangattuparamba, India, 2011.

26. Rehman, A.; Salabun, W.; Faizi, S.; Hussain, M.; Watrobski, J. On Graph Structures in Fuzzy Environment Using Optimization Parameter. IEEE Access 2021, 9, 75699-75711.

27. Somasundaram, A. Domination in fuzzy graphs-II. J. Fuzzy Math. 2005, 13, 281-288.

28. Ni, Y. Fuzzy minimum weight edge covering problem. Appl. Math. Model. 2008, 32, 1327-1337. 
29. Manjusha, O.T.; Sunitha, M.S. Coverings, matchings and paired domination in fuzzy graphs using strong arcs. Iran. J. Fuzzy Syst. 2019, 16, 145-157.

30. Sahoo, S.; Pal, M.; Rashmanlou, H.; Borzooei, R.A. Covering and paired domination in intuitionistic fuzzy graphs. J. Intell. Fuzzy Syst. 2017, 33, 4007-4015.

31. Vinothkumar, N.; Ramya, R. Covering in operations on fuzzy graphs. Int. J. Adv. Sci. Technol. 2019, 28, 1127-1141.

32. Senthilkumar, V.; Ponnappan, C. Note on strong support vertex covering of fuzzy graph by using strong arc. Adv. Appl. Math. Sci. 2019, 18, 1421-1442.

33. Jun, Y.B.; Kim, C.S.; Yang, K.O. Cubic sets. Ann. Fuzzy Math. Inform. 2012, 4, 83-98.

34. Jun, Y.B.; Smarandache, F.; Kim, C.S. Neutrosophic cubic sets. New Math. Nat. Comput. 2017, 13, 41-54.

35. Jun, Y.B.; Song, S.Z.; Kim, S.J. Cubic interval-valued intuitionistic fuzzy sets and their application in BCK/BCI algebras. Axioms 2018, 7, 7 .

36. Jun, Y.B.; Lee, K.J.; Kang, M.S. Cubic structures applied to ideals of BCI-algebras. Comput. Math. Appl. 2011, 62, $3334-3342$.

37. Khan, M.; Jun, Y.B.; Gulistan, M.; Yaqoob, N. The generalized version of Jun's cubic sets in semigroups. J. Intell. Fuzzy Syst. 2015, 28,947-960.

38. Senapati, T.; Jun, Y.B.; Muhiuddin, G.; Shum, K.P. Cubic intuitionistic structures applied to ideals of BCI-algebras. Analele Stiintifice ale Universitatii Ovidius Constanta-Seria Matematica 2019, 27, 213-232.

39. Kang, J.G.; Kim, C.S. Mappings of cubic sets. Commun. Korean Math. Soc. 2016, 31, 423-431.

40. Muhiuddin, G.; Ahn, S.S.; Kim, C.S.; Jun, Y.B. Stable cubic sets. J. Comput. Anal. Appl. 2017, 23, 802-819.

41. Rashid, S.; Yaqoob, N.; Akram, M.; Gulistan, M. Cubic graphs with application. Int. J. Anal. Appl. 2018, 16, 733-750.

42. Muhiuddin, G.; Takallo, M.M.; Jun, Y.B.; Borzooei, R.A. Cubic Graphs and Their Application to a Traffic Flow Problem. Int. J. Comput. Intell. Syst. 2020, 13, 1265-1280.

43. Krishna, K.K.; Rashmanlou, H.; Talebi, A.A.; Mofidnakhaei, F. Regularity of cubic graph with application. J. Indones. Math. Soc. 2019, 25, 1 .

44. Beezer, R.A. Sage for Linear Algebra A Supplement to a First course in Linear Algebra. 2011. Available online: https: //vdocuments.net/sage-for-linear-for-linear-algebra-a-supplement-to-a-first-course-in-linear-algebra.html (accessed on 1 December 2021). 